\title{
Distributed execution of cognitive relaying with time incentive: multiple PU scenario
}

\author{
Taskeen Nadkar ${ }^{1 *}$, Vinay Thumar $^{1}$, Uday B Desai ${ }^{2}$ and Shabbir N Merchant ${ }^{1}$
}

\begin{abstract}
The prime focus of this study is in developing distributed algorithms for cognitive relaying with time incentive for multiple primary users (CRTI-M). CRTI-M is a symbiotic paradigm in which the incumbent primary users (PUs) of the spectrum, with weak transmission links, seek cooperation from the cognitive secondary user (SU) nodes in their vicinity, and in return reward them with an incentive time for the latter's own communication. When relaying through the SU network, each PU can either use its own spectrum or that of the other PUs. Cross-layer optimization problems are formulated to enable both these possibilities in a multi-hop multi-channel cognitive radio network with the objective of maximizing the cumulative time incentive for the SUs. Corresponding distributed algorithms are developed, which face the challenge of meeting the constraints of the formulated problems with only local information and the lack of a centralized controller. Further, to make the CRTI-M schemes practically realizable, a MAC scheduling protocol is suggested, which gives emphasis to the distributed implementation and provides a unified framework for the PUs and SUs. Simulation results are furnished to demonstrate the effectiveness of the proposed algorithms.
\end{abstract}

Keywords: Cognitive radio, Symbiotic cooperative relaying, Cross-layer optimization, Distributed algorithms, OFDM

\section{Introduction}

Cognitive radio $(C R)$ attempts to alleviate the imbalance between spectrum allocation and its use, created by the current command-and-control spectrum access policy. It temporarily allows unused portions of the spectrum (spectrum holes/white-spaces), owned by the licensed users (primary users-PUs), to be accessed by unlicensed users (secondary users-SUs), without causing intrusive interference to the former's communication [1]. This approach can lead to a significant increase in spectrum efficiency, networking efficiency, and energy efficiency [2].

Many schools of thought have evolved from the revolutionary CR paradigm to accommodate substantially different technologies and solutions, one of them being symbiotic cooperative relaying ( $S C R$ ). According to this model, the $P U$ seeks to enhance its own communication by leveraging other users in its vicinity, having better channel conditions, as cooperative relays for its transmission, and in return provides suitable remuneration to them [3-7]. The $S U$ nodes, being scavengers of the

\footnotetext{
*Correspondence: taskeenn@ee.iitb.ac.in

${ }^{1}$ Indian Institute of Technology Bombay, Mumbai 400076, India

Full list of author information is available at the end of the article
}

licensed PU spectrum, are potential candidates as relays. Besides, they have cognitive capabilities, which gives a large amount of flexibility of reconfiguration for resource allocation. The cooperation from the SU network results in enhanced transmission rate of the PU, which translates into reduced transmission time for the same amount of information bits of the PU as those transmitted on its direct link. Then, the time saved can be offered to the SUs for their own communication as a reward for cooperating with the PU (with a fixed rate demand). The SUs can achieve their communication in the time incentive without the need for spectrum sensing. The authors have previously formulated a cross-layer design to enable the $S C R$ scheme called cognitive relaying with time incentive (CRTI), for an orthogonal frequency division multiplexing (OFDM)-based multi-hop CR network [8]. The authors have also proposed that it is possible to reward the SUs with incentive frequency bands, i.e., cognitive relaying with frequency incentive $[7,9]$. While all of the aforementioned works focus on providing centralized solutions to the schemes for a single PU scenario, the main concern of this article is to address a multiple PU model and propose distributed algorithms for the same.

\section{量 Springer}

(c) 2012 Nadkar et al. licensee Springer. This is an Open Access article distributed under the terms of the Creative Commons Attribution License (http://creativecommons.org/licenses/by/2.0), which permits unrestricted use, distribution, and reproduction in any medium, provided the original work is properly cited. 
Some unique challenges are faced when the SCR paradigm is enabled on the spectra of multiple PUs, viz. CRTI for multiple PUs (CRTI-M) [10,11]. The complexity that arises in this scheme as opposed to CRTI for a single PU transceiver is the fact that every PU has its own distinct bandwidth of operation. Consequently, it is crucial to decide the most favorable way in which the available frequency bands be utilized during the relaying process in a multi-hop multi-channel environment. We suggest two methods of CRTI-M: band-restricted relaying (BRR), in which each PU uses its own band when relaying through the SU network, and all-band relaying ( $A B R$ ), in which each PU can use all the available bands (its own, as well as those of the other PUs) when relaying through the SU network. We then formulate cross-layer optimization problems which address the power allocation, frequency domain scheduling, and routing for the two proposed methods, evaluate their upper bound and devise feasible centralized solutions to them.

While the feasible centralized solutions may give reasonably good results in case of a small network, they will require heavy signalling overheads to communicate the global network information to a centralized node in larger CR networks. Also, the computational ability of the node may become a bottleneck. We are thus motivated to explore a solution methodology to the optimization problems for the CRTI-M schemes, viz. $A B R$ and $B R R$, that can be distributedly implemented among the SU nodes. This is the major contribution of the article.

The proposed algorithms address the complexity of distributed resource allocation in a multi-hop multi-channel SU network-an issue which is less commonly addressed in literature. The distinguishing features of the algorithm are as follows: (i) they ensure efficient utilization of the available node power by balancing the throughput along a selected path; (ii) they use a path selection metric which gives due weightage to both the throughput of the complete path as well as that link which may pose a bottleneck for the path; and (iii) incase of a conflict (common resources) between the paths for multiple PUs' sessions, the one which provides the maximum benefit is selected.

Besides, to make the CRTI- $M$ schemes practically realizable, the MAC layer scheduling and physical layer issues are approached in this article with some important concerns: (i) ensuring a completely distributed execution of the schemes among the SU nodes; (ii) developing a unified framework for both the entities (PUs and SUs); and (iii) isolating the PUs from the operation of the SUs, like in the Commons model of CR. However, we do take some liberty of allowing explicit signaling from the PUs to the SUs initially, which happens in the property-rights model (and not in the commons model) [12,13].

The article organization is as follows: (2) section describes the communication scenario and system model.
(3) section presents the centralized problem formulation for both the CRTI-M schemes, viz. BRR and $A B R$. The schemes are compared in (4) section. (5) section provides the details of the proposed distributed algorithms. (6) section explains the utilization of the time incentive by the SUs. (7) section furnishes the details of the distributed implementation and MAC scheduling protocol. (8) section presents simulation results and their detailed analysis. (9) section reports related work, while (10) section concludes the article.

\section{System model}

A CR system with a network of cognitive SUs and multiple PU transceivers is considered (Figure 1a). Each PU has a distinct licensed bandwidth of its own. Normally, the SUs are scavenging all the PUs' spectra for a transmission opportunity. CRTI-M is enabled when one or more PUs, with weak links, seek cooperation from the SU network. We define $\mathbb{Q}$ as the set of PU transceivers participating in the CRTI-M scheme in a specified time frame. OFDM is the communication technology used in the network. We have assumed unit bandwidth for each OFDM sub-carrier. The band-set of each PU is denoted by $\mathbb{M}_{q}, q \in \mathbb{Q}$. Each PU transmitter PU Tx $q$ acts as the source, the receiver $P U R x_{q}$ as the destination, and the SU nodes act as relays in the multi-hop communication (Figure 1b). Decode-and-forward is the relaying technique at each node. The fading gains for various links are mutually independent and are modeled as zero mean complex circular Gaussian random variables. The protocol interference model is assumed [14]. The channel gains are invariant within a frame, but vary over frames (i.e., block-fading channels). We assume that the channel gains from each $P U T x_{q}$ to the SU network, the SU network to $P U R x_{q}$, and those among the SUs, are good enough to provide a significantly higher end-to-end throughput as compared to the direct link of the PU transceiver, resulting in performance gains for both the PUs and SUs.

\section{Problem formulation}

If $C_{\text {textdir }}$ is the throughput obtained on the direct link between the $q$ th PU transceiver, and $C_{\text {textrel }}$ is the maximum throughput achieved when relaying the qth PU's data through the SU network, then the time incentive obtained for the SUs on band-set $\mathbb{M}_{q}$ in a frame duration (normalized to unity) is given by

$$
\lambda_{t_{q}}=1-\frac{C_{\operatorname{dir}_{q}}}{C_{\mathrm{rel}_{q}}} \quad \forall q \in \mathbb{Q}
$$

Ideally, the incentive obtained from each PU $\left(\lambda_{t_{q}}\right)$ should be scaled by its bandwidth $\left(B_{q}\right)$ to effectively capture the incentive obtained, i.e., $\lambda_{t_{q}} B_{q}, q \in \mathbb{Q}$. However, since we have assumed the same bandwidth for all the PUs, 


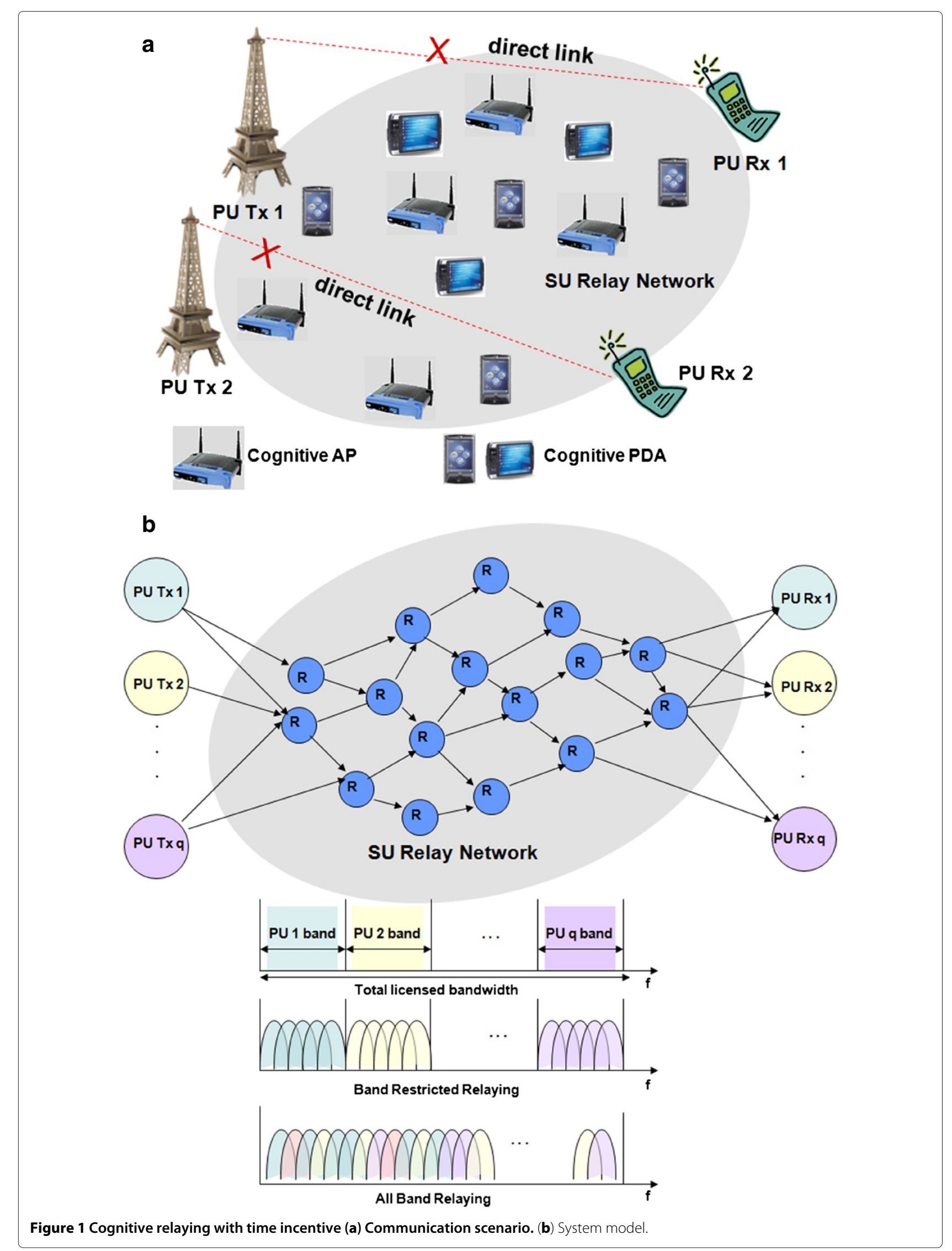


we define the cumulative time incentive as $\sum_{q} \lambda_{t_{q}}$. To efficiently exploit the channel diversities available in the multi-hop multi-channel SU network, we allow flow splitting, and spatial reuse of frequencies outside the interference range of nodes. The optimization problem involves a cross-layer view for power allocation, frequency band scheduling, and routing. A relay with poor channel conditions on all its links will be eliminated from the routes which strive to achieve maximum throughput; thus, relay selection is automatically achieved by the problem. In the following sections, two schemes for CRTI-M are proposed.

\subsection{BRR}

In the first $C R T I-M$ scheme, referred to as BRR, the data from each PU are relayed on its respective frequency band-set $\mathbb{M}_{q}$ within the SU network (Figure 1b). If we define the communication between each unique participating PU transceiver, i.e., PU $T x_{q}-\mathrm{PU} R x_{q}, q \in \mathbb{Q}$, as a session, the problem entails solving a multi-session optimization with the objective of maximizing the cumulative time incentive, i.e., $\sum_{q} \lambda_{t_{q}}$. Since each PU communicates on its own band within the SU network, the sessions can be thought of as independent problems; hence the variables $x_{i j}^{m}, P_{i j}^{m}$, and $f_{i j}$ (which denote the band assignment, power allocation, and flow, respectively) are indexed by the session number. These problems are, however, connected by the node power and interference constraints.

Optimization problem (P1):

$$
\max _{\left(x_{i j}^{m}(q), P_{i j}^{m}(q), f_{i j}(q)\right)} \sum_{q \in \mathbb{Q}} \lambda t_{q}
$$

$\lambda_{t_{q}}$ is as defined in (1), in which $C_{\text {rel }_{q}}$ is given by

$$
C_{\text {rel }_{q}}=\sum_{j \in T_{i}} f_{i j}(q) \quad \forall q \in \mathbb{Q}, \quad i=\operatorname{PU~} T x_{q}
$$

Flow constraints:

$$
\begin{aligned}
& \sum_{j \in T_{i}}^{j \neq \mathrm{PUTx}_{q}} f_{i j}(q)=\sum_{k \in T_{i}}^{k \neq \mathrm{PUR} x_{q}} f_{k i}(q) \forall i \in \mathbb{N}, q \in \mathbb{Q}, i \neq \mathrm{PU} T x_{q}, R x_{q} \\
& f_{i j}(q) \geq 0 \quad \forall(i, j) \in \mathbb{E}, \quad \forall q \in \mathbb{Q} \\
& f_{i j}(q)-\sum_{m \in \mathbb{M}_{q}} \log _{2}\left(1+\frac{h_{i j}^{m} P_{i j}^{m}(q)}{\sigma^{2}}\right) \leq 0 \quad \forall(i, j) \in \mathbb{E}, q \in \mathbb{Q}
\end{aligned}
$$

We assume bidirectional links; each node $i$ in the graph has a transmit/receive set of nodes $T_{i}$. $f_{i j}(q)$ is the data flow (bits/s) from node $i$ to node $j$ for the $q$ th session. Equation (4) indicates that, except for the source (PU $T x_{q}$ ) and destination (PU $R x_{q}$ ) nodes, the inflow into a node is equal to the outflow. Equation (5) ensures that all the flows are non-negative. Equation (6) refers to the fact that the flows on a link cannot exceed its capacity according to Shannon's channel capacity theorem [15]. $h_{i j}^{m}$ denotes the channel power gain on band $m$ and $P_{i j}^{m}(q)$ denotes the corresponding power allocation for the $q$ th session. In (6), the $\log$ function contains only $\sigma^{2}$ in the denominator due to the use of an interference model, which ensures that when node $i$ is transmitting to node $j$ on band $m$, the interference from all other nodes in this band must remain negligible due to the frequency domain scheduling and interference constraints. $\mathbb{N}$ denotes the node set of the network (including PU $T x_{q}, \mathrm{PU} R x_{q}, \forall q \in \mathbb{Q}$ and the SUs), and $\mathbb{E}$ denotes the edge set.

Frequency Domain Scheduling Constraints:

$$
\sum_{j \in T_{i}} x_{i j}^{m}(q)+\sum_{k \in T_{i}} x_{k i}^{m}(q) \leq 1 \quad \forall i \in \mathbb{N}, m \in \mathbb{M}_{q}, q \in \mathbb{Q}
$$

Equation (7) suggests that if a node $i$ has used a band $m$ for transmission or reception, it cannot be used by node $i$ again for any other transmission or reception. $x_{i j}^{m}(q)$ is a binary variable which takes the value 1 if and only if band $m \in \mathbb{M}_{q}$ is active on link $(i, j)$, i.e.,

$$
x_{i j}^{m}(q)=\{0,1\} \quad \forall(i, j) \in \mathbb{E}, \quad m \in \mathbb{M}_{q}, \quad q \in \mathbb{Q}
$$

Power constraints:

$$
P_{i j}^{m}(q)-P_{T_{i j}}^{m} x_{i j}^{m}(q) \geq 0 \quad \forall(i, j) \in \mathbb{E}, \quad m \in \mathbb{M}_{q}, \quad q \in \mathbb{Q}
$$

$$
P_{i j}^{m}(q)-P_{\text {peak }} x_{i j}^{m}(q) \leq 0 \quad \forall(i, j) \in \mathbb{E}, \quad m \in \mathbb{M}_{q}, \quad q \in \mathbb{Q}
$$

$\sum_{q \in \mathbb{Q}} \sum_{j \in T_{i}, m \in \mathbb{M}_{q}} P_{i j}^{m}(q) \leq P_{\text {node }_{i}} \quad \forall i \in \mathbb{N}$

Equations (9) and (10) ensure that $P_{i j}^{m}(q) \in\left[P_{T_{i j}}^{m}, P_{\text {peak }}\right]$ if the band $m$ is selected, and $P_{i j}^{m}(q)=0$ if the band is not selected. The data transmission from node $i$ to $j$ is successful only if the received transmission power exceeds a power threshold $P_{T}$, from which we can calculate the minimum required transmission power on a band $m$ at node $i$ as $P_{T_{i j}}^{m}=P_{T} / h_{i j}^{m}$. $P_{\text {peak }}$ denotes the maximum power that can be allocated to any band $m$. Equation (11) ensures that the power consumed at node $i$ for all the sessions over all the bands cannot exceed the node power $P_{\text {node }_{i}}$. 
Interference constraints:

$$
\begin{aligned}
P_{k h}^{m}(q)+ & \left(\sum_{k \in I_{j}^{m}} \sum_{q \in \mathbb{Q}} P_{k h}^{m}(q) h_{k j}^{m}\right. \\
& \left.-P_{I}+P_{\text {peak }}-P_{k h}^{m}(q)\right) x_{i j}^{m}(q) \leq P_{\text {peak }}
\end{aligned}
$$

$\forall i \in \mathbb{N}, m \in \mathbb{M}_{q}, q \in \mathbb{Q}, j \in T_{i}, k \in I_{j}^{m}, k \neq i$

Equation (12) ensures that for a successful transmission on link $i$ to $j$, on an interfering link $k$ to $h$, the transmit power on any band $m$ cannot exceed $P_{\text {peak }}$ if $x_{i j}^{m}(q)=0$; if $x_{i j}^{m}(q)=1$ then the total interference received by node $j$ on band $m$ cannot exceed the interference threshold $P_{I}$.

Upper bound and a feasible centralized solution:

In the above formulation $h_{i j}^{m}, \sigma^{2}, P_{T}, P_{I}, P_{\text {peak }}, P_{\text {node }_{i}}$ are all constants, while $x_{i j}^{m}(q), P_{i j}^{m}(q), f_{i j}(q)$ are the optimization variables. It is clearly a mixed integer non-linear programming problem. Based on the discussion on similar problems in $[14,16]$ and the references therein, we conjecture that the given problem is NP-hard. A linear relaxation of the $\log$ term by the use of tangential supports [9] will drastically simplify the formulation and will provide an upper bound to the original problem solution. Also, a feasible sub-optimum solution can be obtained by decoupling the operations of power allocation and band scheduling, and that of flow computation, as follows:

(1) The power allocation and band scheduling $\left(P_{i j}^{m}(q), x_{i j}^{m}(q)\right)$ are obtained from the log relaxed problem with tangential supports. This solution, however, may violate the flow constraints.

(2) The above $\left(P_{i j}^{m}(q), x_{i j}^{m}(q)\right)$ are substituted in the original problem, which is then solved only with respect to $f_{i j}(q)$ as the optimization variable. The overall result represents a feasible solution to the original problem $P 1$.

\section{$3.2 \quad A B R$}

In the second scheme of CRTI-M called $A B R$, the data from each PU can be relayed on the complete frequency band available within the SU network (which is the union of the band-sets of the participating PUs). This is unlike $B R R$, where the PU's data are relayed only on its own bandset. The data from the various PUs are thus frequency interleaved on all available bands within the SU network in $A B R$ (Figure 1b).

The objective is to maximize the cumulative time incentive obtained from all the PUs. Though majority of the problem formulation for $A B R$ is similar to that for $B R R$, the main difference is in the fact that in the latter the constraints have to be posed for each PU's band-set, while in the former the constraints are posed on the complete available band-set.

Optimization problem (P2):

$$
\max _{\left(x_{i j}^{m}(q), P_{i j}^{m}(q), f_{i j}(q)\right)} \sum_{q \in \mathbb{Q}} \lambda_{t_{q}}
$$

Flow constraints:

$$
\sum_{j \in T_{i}}^{j \neq \text { PUT }_{q}} f_{i j}(q)=\sum_{k \in T_{i}}^{k \neq \text { PUR } x_{q}} f_{k i}(q) \forall i \in \mathbb{N}, q \in \mathbb{Q}, i \neq \text { PUTx }_{q}, R x_{q}
$$

$f_{i j}(q) \geq 0 \quad \forall(i, j) \in \mathbb{E}, \quad \forall q \in \mathbb{Q}$

$f_{i j}(q)-\sum_{m \in \mathbb{M}} \log _{2}\left(1+\frac{h_{i j}^{m} P_{i j}^{m}(q)}{\sigma^{2}}\right) \leq 0 \quad \forall(i, j) \in \mathbb{E}, q \in \mathbb{Q}$

In (16),

$$
\mathbb{M}= \begin{cases}\mathbb{M}_{q} & \text { if } i=\mathrm{PU} T x_{q} O R j=\mathrm{PU} R x_{q} \\ \hat{\mathbb{M}} & \text { otherwise. }\end{cases}
$$

in which $\hat{\mathbb{M}}=\bigcup_{q \in \mathbb{Q}} \mathbb{M}_{q}$. Equation (16) indicates that the sum of the flows of all sessions on a link cannot exceed the capacity of a link according to Shannon's channel capacity theorem. Furthermore, Equation (17) describes the fact that only the PU's own frequency band-set $\mathbb{M}_{q}$ will be available on the first and last hops, i.e., PU $T x_{q}$ to the SU network and SU network to PU $R x_{q}$, while within the SU network, the total bandwidth of all the participating PUs is available.

The rest of the constraints, i.e., frequency domain scheduling, power, and interference constraints, are the same as those posed for $B R R$, with $\mathbb{M}_{q}$ replaced by $\mathbb{M}$ (17). However, an additional frequency domain scheduling constraint is introduced to ensure that a band on a given edge is not used by more than one PU. There are two reasons for incorporating this constraint: (i) It results in a much simpler formulation than if multiple PUs were allowed to use the same band; and (ii) it allows a different QoS parameter to be set for each PU if desired.

$$
\sum_{q \in \mathbb{Q}} x_{i j}^{m}(q) \leq 1 \quad \forall(i, j) \in \mathbb{E}, m \in \mathbb{M}
$$

Note: In the ABR problem formulation, it is possible to allow multiple sessions to use the same band rather simply (thereby eliminating constraint (18)), without separating the power for the sessions as $P_{i j}^{m}(q), q \in \mathbb{Q}$. However, when the SUs are rewarded with the incentive time on each PU's band, it is possible that they utilize it 'selfishly', i.e., in proportion to the power spent in relaying each PUs data. Given this situation, the above formulation seems the most appropriate. 
The nature of problem $P 1$ is the same as that of $P 2$, and consequently, the upper bound and feasible centralized solution are computed in the same way as $B R R$.

\section{Comparing BRR and ABR}

As described earlier, $A B R$ allows the data from each PU to be relayed on frequency bands which do not necessarily belong to its own band-set, providing more channel diversity and consequently higher end-to-end throughput for the PUs, as compared to $B R R$. BRR, however, has one distinct advantage over $A B R$-if interference management has to be done for the individual PUs (this may be needed in case of PU prioritization, critical data transmission, or stringent QoS requirements), it is much easier in $B R R$ since the PUs are always communicating on their own band-set, even within the SU network. The band-set utilized by each PU is contiguous and is known a priori, unlike $A B R$, wherein the data from the different PUs are interleaved on all available frequency bands.

\section{Distributed algorithms}

Motivated by the need to diminish the communication overheads of transferring the network information to a centralized controller (which may also have limited processing power) to solve the optimization problems for $B R R$ and $A B R$, in this section we devise distributed algorithms for the schemes.

\subsection{Distributed BRR}

In $B R R$, only the PUs' own band-set $\mathbb{M}_{q}$ is available within the SU network while trying to maximize the cumulative time incentive.

The algorithm comprises the following modules: Initialization, Cost Computation, Path Selection, Band Assignment, Power Allocation, Update Allocation, and Flow Computation. Each module operates on information locally available at the SU node for its computations. First, we describe the details of each of the modules, and then explain how the modules interact in the distributed algorithm for $B R R$.

Initialization:

$$
\begin{aligned}
& \text { For all } i \in \mathbb{N} \\
& \text { For all }(i, j) \in \mathbb{E} \\
& \text { For all } m \in \mathbb{M}_{q} \\
& \begin{array}{l}
x_{i j}^{m}=0, P_{i j}^{m}=0 \\
P^{m}=P_{\text {peak }}
\end{array} \\
& P_{T_{i j}}^{m}=P_{T} / h_{i j}^{m} \\
& \text { bcost }_{i j}^{m}=0
\end{aligned}
$$

End For

End For

End For
In this module, the band allocation $x_{i j}^{m}$ and power allocation $P_{i j}^{m}$ are initialized to 0 . The peak power $P_{\text {peak }_{i j}}^{m}$ is initially assigned a fixed value $P_{\text {peak }}$, but will be later updated based on the interference constraints. The detection threshold $P_{T_{i j}}^{m}$ is computed and bcost ${ }_{i j}^{m}$ is initialized to 0 .

\section{Cost Computation:}

$\%$ Computing bcost

For all $i \in \mathbb{N}$

$$
\begin{aligned}
& \text { For all }(i, j) \in \mathbb{E} \\
& \text { For all } m \in \mathbb{M}_{q} \\
& \text { If } \mathrm{bcost}_{i j}^{m}=\infty \\
& \text { continue } \\
& \text { Else } \\
& P o_{i j}^{m}=0, x o_{i j}^{m}=0 \\
& P_{\text {sum }}(i)=\sum_{j \in T_{i}} \sum_{m \in \mathbb{M}_{q}} x_{i j}^{m} P_{i j}^{m} \\
& \text { If } P_{T_{i j}}^{m} \geq P_{\text {peak }_{i j}}^{m} \\
& \text { Else } \\
& \mathrm{bcost}_{i j}^{m}=\infty \\
& \text { If } x_{i j}^{m}==0 \\
& p w r_{i j}^{m}=\min \left(P_{\text {peak }_{i j}}^{m}, P_{\text {node }_{i}}-P_{\text {sum }}(i)\right) \\
& \text { If } p w r_{i j}^{m} \geq P_{T_{i j}}^{m} \\
& \operatorname{bcost}_{i j}^{m}=\frac{1}{\log _{2}\left(1+\frac{p w r_{i j}^{m} h_{i j}^{m}}{\sigma^{2}}\right)} \\
& \text { bcost }_{i j}^{m}=\infty \\
& \text { If } P_{i j}^{m}<P_{\text {peak }_{i j}}^{m} \\
& p w r_{i j}^{m}=\min \left(P_{\text {peak }_{i j}}^{m}-P_{i j}^{m}, P_{\text {node }_{i}}-P_{\sum}(i)\right) \\
& \mathrm{bcost}_{i j}^{m}=\frac{1}{\log _{2}\left(1+\frac{\left(p w w_{i j}^{m}+P_{i j}^{m}\right) h_{i j}^{m}}{\sigma^{2}}\right)-\log _{2}\left(1+\frac{P_{i j}^{m} h_{i j}^{m}}{\sigma^{2}}\right)} \\
& \text { bcost }_{i j}^{m}=\infty \\
& \text { End If }
\end{aligned}
$$

\section{End If}

\section{End if}

End For

End For

End For

$\%$ Computing lcost

For all $i \in \mathbb{N}$

For all $(i, j) \in \mathbb{E}$

End For

$$
\operatorname{lcost}_{i j}=\min _{m \in \mathbb{M}_{q}}\left(\mathrm{bcost}_{i j}^{m}\right)
$$

End For 
In Cost computation, we define two costs which will be used in the path selection metric: the band cost (bcost) and the link cost (lcost). The bcost is the inverse of the throughput that can be achieved from the band. The lcost is the least $b$ cost from among all the bands on a link. Those bands whose detection threshold exceeds the peak power constraint are removed by making the bcost infinite. The computation of the bcost is different in the two events: in case of a new allocation, the bcost is based on the peak power (limited by the available node power); in case of an already allocated band, it depends on the difference between the allocated power and peak power (limited by the available node power).

\section{Path Selection:}

The Distributed Dijkstra's Algorithm [17] is used to compute the least cost path on $\mathbb{M}_{q}$ from the PU Tx $q$ to the PUR $x_{q}$, using the metric:

Met $=w_{1} * \sum_{(i, j) \in \text { path }}$ lcost $_{i j}+w_{2} * \max _{(i, j) \in \text { path }}$ lcost $_{i j}$ If no path is found by the algorithm, found $=0$.

$w_{1}$ and $w_{2}$ are the weights assigned in the metric to give the desired importance to the sum of the lcosts and the bottleneck link (which is determined by $\max _{(i, j) \in \text { path }}$ lcost $\left._{i j}\right)$. Following the path selection, in every iteration the band assignment module assigns frequency bands on the selected path. The algorithm exits the current iteration when no path can be found by path selection.

Band assignment: \% Assigning the band on each link on the path

For all $(i, j) \in \mathbb{L}_{\text {set }}$ (in order from source to destination)

$$
\begin{gathered}
m 1(i, j)=\arg \min _{m \in \mathbb{M}_{i j}}\left(\text { bcost }_{i j}^{m}\right) \\
\mathbb{M}_{i j}=\mathbb{M}_{i j} \backslash m 1(i, j) \\
m 2(i, j)=\arg \min _{m \in \mathbb{M}_{i j}}\left(\text { bcost }_{i j}^{m}\right) \\
\text { If } m 1(i, j) \neq m 1(j, k) \\
x o_{i j}^{m 1}=x o_{j k}^{m 1}=1 \\
\text { Else }
\end{gathered}
$$

$$
\begin{aligned}
& \text { If } \text { bcost }_{i j}^{m 2}=\infty A N D \text { bcost }_{j k}^{m 2}=\infty \\
& \text { bcost }_{i j}^{m 1}=\text { bcost }_{j k}^{m 1}=\infty \\
& \text { revoke }=1 \\
& \text { Goto Path Selection } \\
& \text { Else } \\
& \text { If } \\
& \begin{array}{c}
\left(\mathrm{bcost}_{i j}^{m 2}-\mathrm{bcost}_{i j}^{m 1}\right) \geq\left(\mathrm{bcost}_{j k}^{m 2}-\mathrm{bcost}_{j k}^{m 1}\right) \\
x o_{i j}^{m 1}=x o_{j k}^{m 2}=1
\end{array} \\
& \text { End If } \\
& x o_{j k}^{m 1}=x o_{i j}^{m 2}=1 \\
& \text { End If }
\end{aligned}
$$

End For
$\%$ Ensuring that the allocated band is not used again for transmission or reception

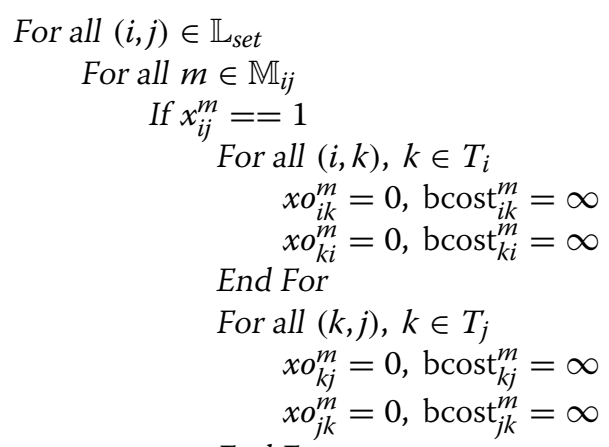

End If

End For

End For

The Band assignment module consists of two main parts: assigning a band on each link of the selected path, and ensuring that the band assigned is not used again by the same node for transmission or reception (frequency domain scheduling constraints). For allocation, the band with the least bcost is preferred. $\mathbb{L}_{\text {set }}$ represents the linkset of the path under consideration, in order from source to destination. $\mathbb{M}_{i j}$ is the band-set on link $(i, j)$ on the current path. In case of a conflict on adjacent links, the band with the next highest bcost is chosen. If that is not possible, then the current path is revoked, and the path selection module is used to compute a new path. It should be noted that the variables $x o_{i k}^{m}$ and $P o_{i k}^{m}$ are used to denote the band assignment and power allocation, respectively, in the current iteration, while $x_{i k}^{m}$ and $P_{i k}^{m}$ denote the overall allocation. When a path is not found by the path selection module, the flag found is reset and the variable mcap is assigned zero. The flag revoke is reset if a suitable path cannot be found without violating the frequency domain scheduling constraints by band assignment.

\section{Power allocation: \\ \% Computing residual capacity}

$$
\begin{aligned}
& \text { For all }(i, j) \in \mathbb{L}_{s e t} \\
& \text { For all } m \in \mathbb{M}_{i j} \\
& \text { If } x_{i j}^{m}==0 A N D x o_{i j}^{m}==1 \\
& \text { Else }{ }^{c a p_{i j}^{m}}=\log _{2}\left(1+\frac{p w r_{i j}^{m} h_{i j}^{m}}{\sigma^{2}}\right) \\
& \text { If } x_{i j}^{m}==1 A N D x o_{i j}^{m}==1 \\
& \operatorname{cap}_{i j}^{m}=\log _{i j}^{m}=\log _{2}\left(1+\frac{P_{i j}^{m} h_{i j}^{m}}{\sigma^{2}}\right) \\
& \text { End If }
\end{aligned}
$$




\section{End For}

\section{End For}

$\%$ Balancing the capacity along the path

$$
\begin{aligned}
& m c a p=\min _{(i, j) \in \mathbb{L}_{\text {set }}} \text { cap } p_{i j}^{m} \\
& \left(i^{\prime}, j^{\prime}\right)=\arg \min _{(i, j) \in \mathbb{L}_{\text {set }}} \text { cap } p_{i j}^{m} \\
& \mathbb{L}_{\text {set }}=\mathbb{L}_{\text {set }} \backslash\left(i^{\prime}, j^{\prime}\right)
\end{aligned}
$$

$\%$ Allocating power

$$
\begin{aligned}
& \text { For all }(i, j) \in \mathbb{L}_{\text {set }} \\
& \text { For all } m \in \mathbb{M}_{i j} \\
& \text { If } x_{i j}^{m}==0 \text { AND } x o_{i j}^{m}==1 \\
& P o_{i j}^{m}=\left(2^{m c a p}-1\right) \frac{\sigma^{2}}{h_{i j}^{m}} \\
& \text { If } P o_{i j}^{m}<P_{T_{i j}}^{m} \\
& \text { End If } \\
& P o_{i j}^{m}=P_{T_{i j}}^{m} \\
& \text { If } x_{i j}^{m}==1 A N D x o_{i j}^{m}==1 \\
& P o_{i j}^{m}=\left(2^{m c a p+c o_{i j}^{m}}-1\right) \frac{\sigma^{2}}{h_{i j}^{m}}-P_{i j}^{m} \\
& \text { End If }
\end{aligned}
$$

\section{End For}

$\%$ Updating peak power in view of the interference constraints

$$
\begin{aligned}
& \text { For all } j \in \mathbb{N}_{\text {set }} \backslash P U T x \\
& \text { For all } m \in \mathbb{M}_{i j} \\
& \text { If } x o_{i j}^{m}==1 \\
& \text { For all } k \in T_{j} \backslash \mathbb{N}_{\text {set }}, l \in T_{k}, l \neq j \\
& P_{\text {peak }}^{m}=\frac{P_{I}-i n t_{j}}{h_{k j}^{m}} \\
& \text { End For } \\
& \text { End If }
\end{aligned}
$$

\section{End For}

The main objective of the power allocation module is to allocate the power based on the least capacity from among all the links of the selected path (mcap). We first compute the capacity based on the current allocation of bands and power constraints. We refer to it as the residual capacity $\left(\operatorname{cap}_{i j}^{m}\right)$. This is because, in case the band is already allocated, it is a difference of the capacity based on the previous allocation and the current allocation. Besides balancing the capacities, the module ensures that the power constraints and interference constraints described in the centralized problem are met. In the module, $\mathbb{N}_{\text {set }}$ denotes the node-set of the path under consideration, and $i n t_{j}$ is the measured interference power at node $j$.

The entire algorithm with the interactions between its modules is depicted in the flow chart of Figure 2. In each iteration, a source-destination pair (PU $T x_{q}-\mathrm{PU} R x_{q}, q \in$ $\mathbb{Q})$ is selected in a round-robin manner. For each PU, the Initialization, Cost Computation, Path Selection, Band Assignment, Power Allocation modules are executed to obtain a single path with the corresponding power allocation and band assignment. The power allocation and band assignment are stored but not fixed. Then the stored assignments for each PU are compared on the basis of the minimum capacity along the path $(\operatorname{mcap}(q))$. The power allocation and band assignment for the PU $T x_{q}-$ PU $R x_{q}$ pair with the highest ratio of $m c a p(q)$ to $C_{\operatorname{dir}_{q}}$ is fixed, while the other assignments are revoked. Based on this assignment, the update allocation module is executed. The overall band and power allocation $\left(x_{i j}^{m}, P_{i j}^{m}\right)$ are updated with that of the current iteration $\left(x o_{i j}^{m}, P o_{i j}^{m}\right)$.

\section{Update allocation:}

$\% q^{\prime}$ represents the selected session

$$
\begin{aligned}
& \text { For all } i \in \mathbb{N} \\
& \qquad \begin{array}{c}
\text { For all }(i, j) \in \mathbb{E} \\
\qquad \begin{array}{c}
\text { For all } m \in \mathbb{M}_{q} \\
P_{i j}^{m}=P_{i j}^{m}+\operatorname{Po}_{i j}^{m}\left(q^{\prime}\right) \\
x_{i j}^{m}=x_{i j}^{m}
\end{array} \text { OR xoo } o_{i j}^{m}\left(q^{\prime}\right) \\
\text { End For }
\end{array}
\end{aligned}
$$

End For

\section{End For}

When no paths are found for any of the sessions in a given iteration, the algorithm terminates and computes the flow.

\section{Flow computation:}

The Ford-Fulkerson algorithm [18] is used to compute the maximum network flow originating from the PU $T x_{q}, q \in \mathbb{Q}$.

Note: What is described above is what happens when the algorithm is programmed. As far as the practical distributed implementation is concerned, the SUs will simultaneously compute the assignment for all PU T $x_{q}-P U R x_{q}$ pairs. In case the path belonging to two or more pairs encounters a common node, there is a conflict in the resource allocation. In such a situation, the common node, which will have knowledge of the 'mcaps' of the paths passing through it, will allocate the path of that PU $T x_{q}-$ $P U R x_{q}$ pair with the 'highest ratio of $m c a p(q)$ to $C_{d i r_{q}}$ ', while the others will be revoked.

\subsection{Distributed ABR}

The distributed algorithm for $A B R$ involves the same modules as $B R R$, and operates in a similar manner as the latter. However, an important difference is that in $A B R$, 


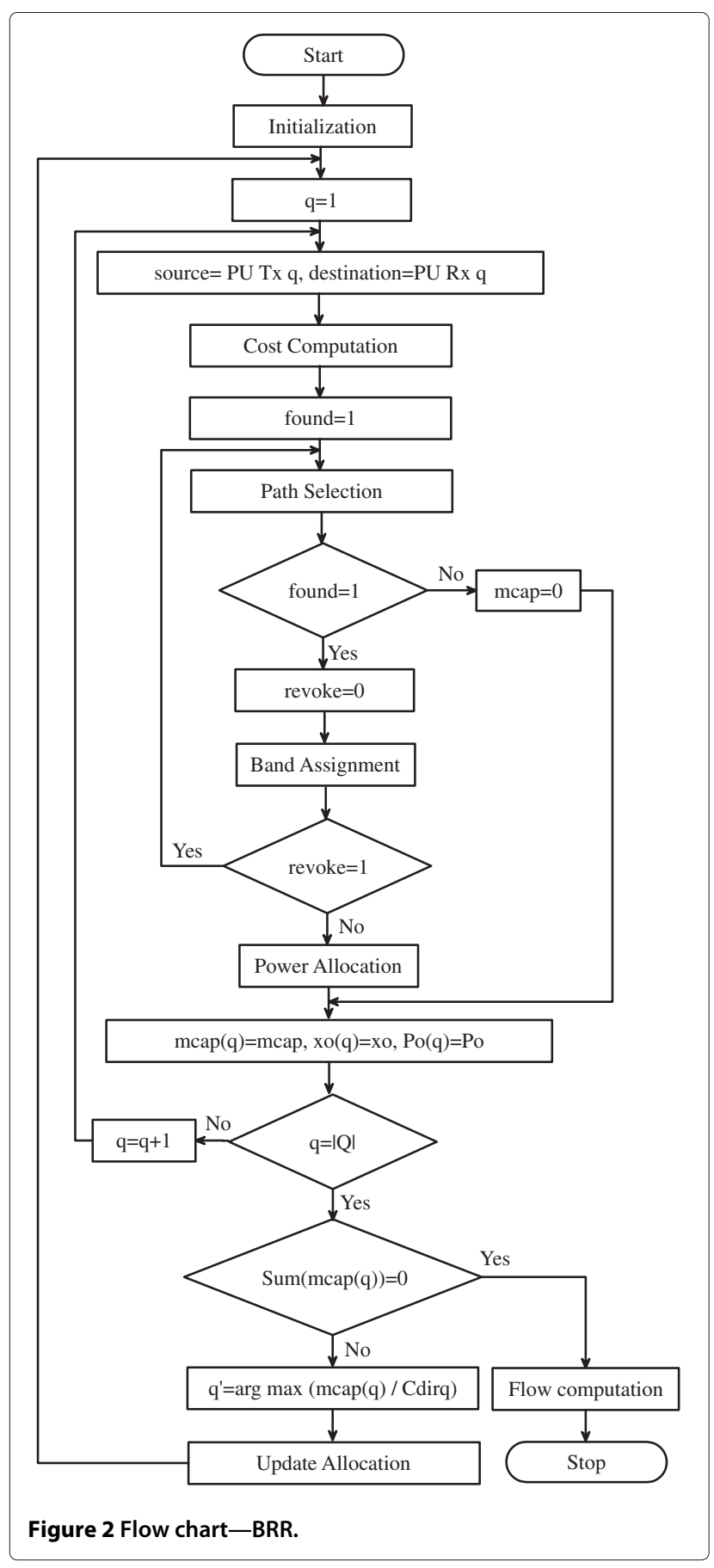

the total band-set of all the participating PUs, i.e., $\hat{\mathbb{M}}=$ $\bigcup_{q \in \mathbb{Q}} \mathbb{M}_{q}$, is available within the SU network, while only the PU's own frequency band-set $\mathbb{M}_{q}$ is available on the first and last hops, i.e., PU $T x_{q}$ to the SU network and SU network to PU $R x_{q}$.

Another difference in the distributed algorithm for $A B R$ is the requirement of keeping the various PU's sessions on distinct bands (as described in the corresponding centralized problem $P 2$ ). For this purpose, an additional module

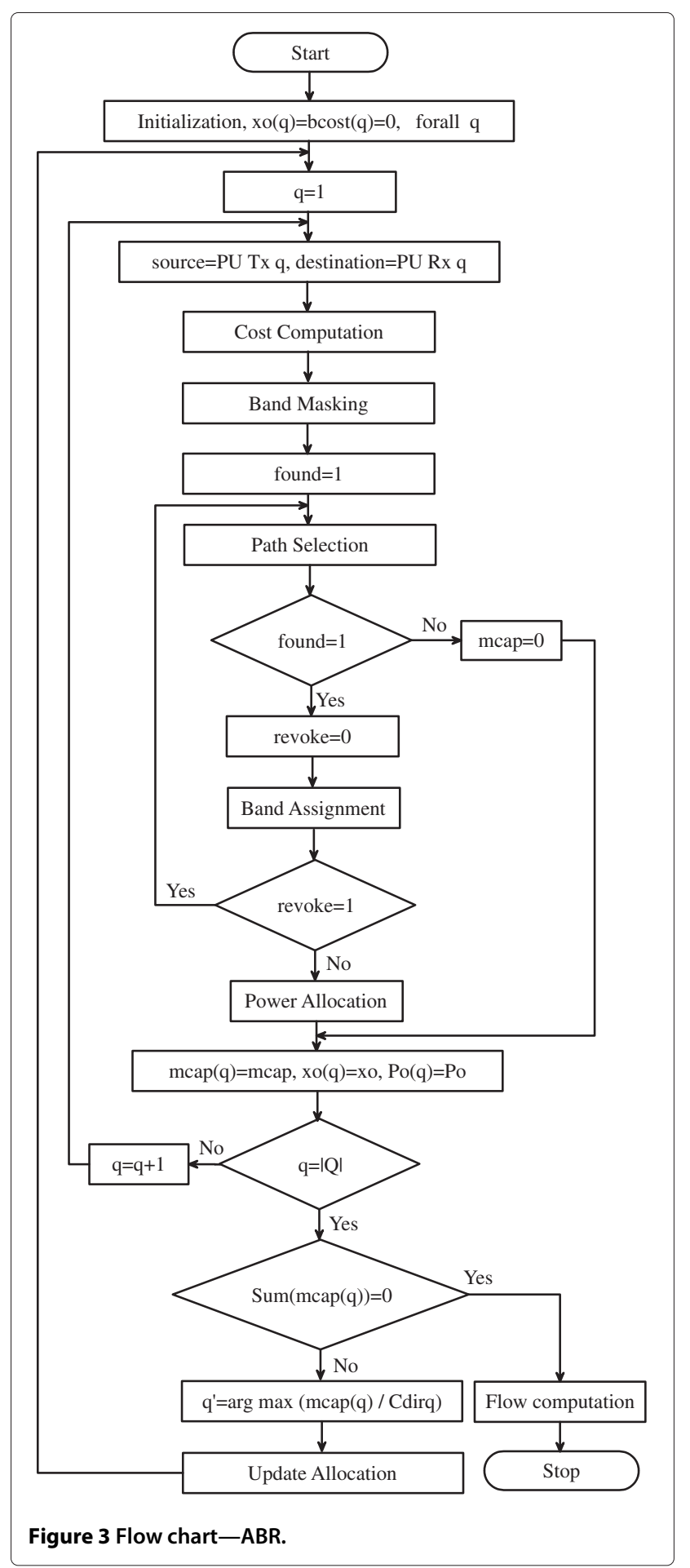

called Band Masking is incorporated. The details of the module are as follows.

\section{Band masking:}

For all $p \in \mathbb{Q}, p \neq q$ For all $i \in \mathbb{N}$ 
For all $(i, j) \in \mathbb{E}$

For all $m \in \mathbb{M}$

$$
\begin{aligned}
& \text { If } x_{i j}^{m}(p)==1 \\
& \quad \mathrm{bcost}_{i j}^{m}=\infty \\
& \text { End If }
\end{aligned}
$$

End For

End For

End For

End For

For $p=q, p \in \mathbb{Q}$

For all $i \in \mathbb{N}$

For all $(i, j) \in \mathbb{E}$

For all $m \in \mathbb{M}$

$$
\begin{aligned}
& \text { If } x_{i j}^{m}(p)==1 \\
& \quad \mathrm{bcost}_{i j}^{m}=\mathrm{bcost}_{i j}^{m}(p)
\end{aligned}
$$$$
\text { End If }
$$

End For

End For

End For

End For

When executing the iteration for a certain PU $T x_{q}-$ PU $R x_{q}$ pair, the bands which have been utilized so far for all the sessions except the current session are masked by setting their bcost to infinity. It prevents the same band from being used by more than one session. Besides that, the update allocation module is modified accordingly.

\section{Update allocation:}

$\% q^{\prime}$ represents the selected session

$$
\begin{aligned}
& \text { For all } i \in \mathbb{N} \\
& \text { For all }(i, j) \in \mathbb{E} \\
& \text { For all } m \in \mathbb{M} \\
& \begin{array}{l}
P_{i j}^{m}=P_{i j}^{m}+P o_{i j}^{m}\left(q^{\prime}\right) \\
x_{i j}^{m}\left(q^{\prime}\right)=x_{i j}^{m}\left(q^{\prime}\right) \text { OR } x o_{i j}^{m}\left(q^{\prime}\right) \\
x_{i j}^{m}=x_{i j}^{m} \text { OR } x o_{i j}^{m}\left(q^{\prime}\right) \\
\operatorname{bcost}_{i j}^{m}\left(q^{\prime}\right)=\operatorname{bcost}_{i j}^{m}
\end{array}
\end{aligned}
$$

End For

End For

The entire algorithm with the interactions between its various modules is depicted in the flow chart of Figure 3.

\section{Utilization of the time incentive}

It was discussed in the previous sections that in the $C R T I-M$ scheme (deploying $B R R$ or $A B R$ ), each PU takes assistance from the multi-hop network of SUs to relay its data when the link between its transmitter and receiver is

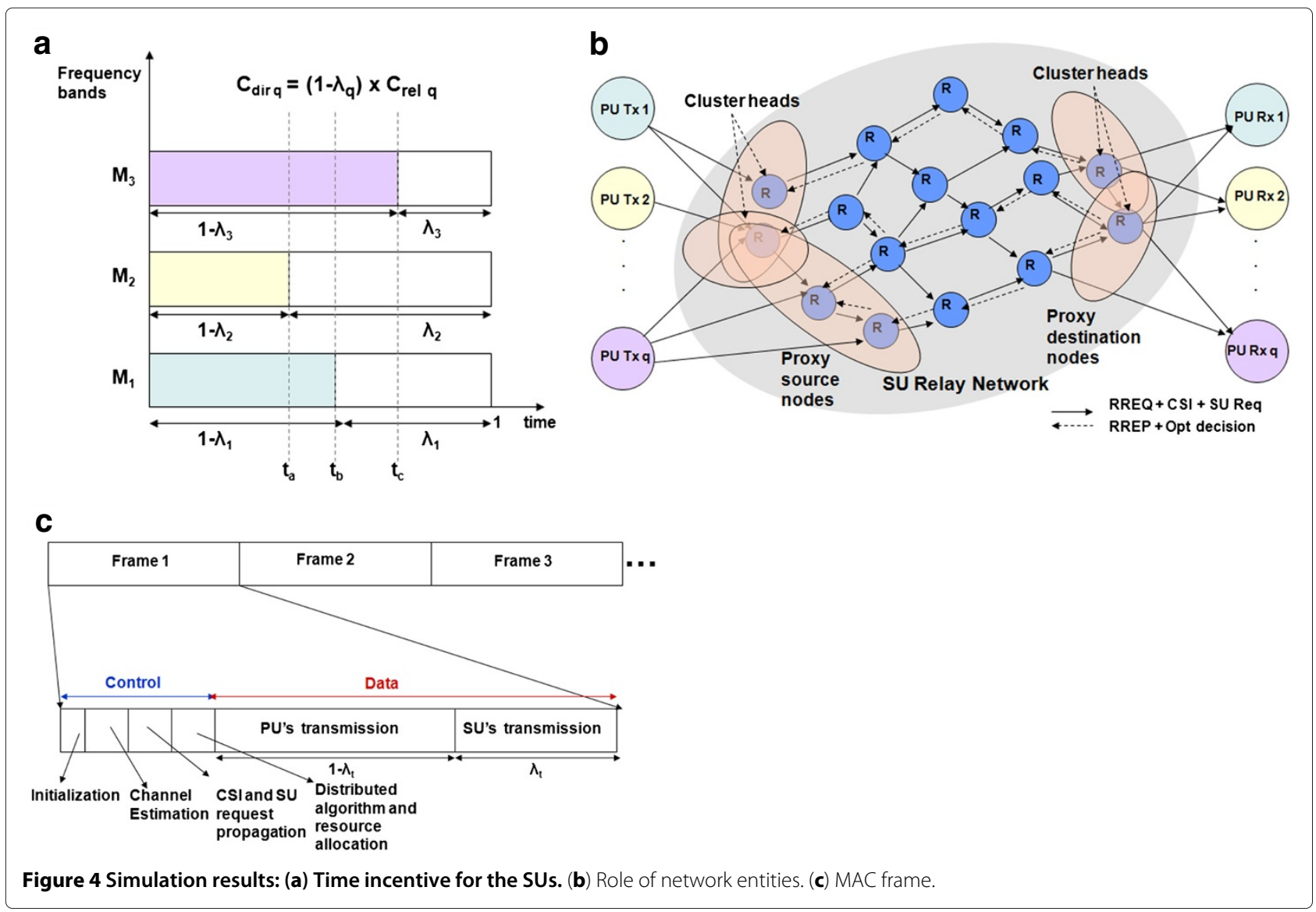


weak. In return, the PU rewards them with an incentive time. As defined earlier, $C_{\operatorname{dir}_{q}}$ is the throughput obtained by using the weak link between PU $T x_{q}$ and PU $R x_{q}$, $q \in \mathbb{Q}$, and $C_{\mathrm{rel}_{q}}$ is the maximum throughput obtained when relaying the $q$ th PU's data through the SU network. If $C_{\operatorname{dir}_{q}}=\left(1-\lambda_{t_{q}}\right) C_{\mathrm{rel}_{q}}, 0 \leq \lambda_{t_{q}} \leq 1$, then $\lambda_{t_{q}}$ is the time incentive obtained in a unit time slot on the band-set $\mathbb{M}_{q}$.

It can be observed from Figure 4a that the time incentive that will be obtained for the SUs may be different on the different PUs' bands, depending on $C_{\operatorname{dir}_{q}}$ and $C_{\mathrm{rel}_{q}}$. For instance, in $\left(t_{a}-t_{b}\right)$ only $\mathbb{M}_{2}$ is available, while in $\left(t_{b}-t_{c}\right)$ $\mathbb{M}_{1} \cup \mathbb{M}_{2}$ is available. For each such interval $\left(t_{x}-t_{y}\right)$ in a frame, on the available band-set $\mathbb{M}_{x y}$, a multi-session sum throughput maximization problem can be solved for the SUs to efficiently exploit the transmission opportunity created by CRTI-M.

\section{Protocol design}

\subsection{Physical layer considerations}

The execution of the distributed algorithms assumes the knowledge of accurate channel state information (CSI) at the nodes. The estimation of local CSI is done by measuring the received power of the pilot signals. Discontiguous OFDM is used for data transmission, which allows the relays to decode only a fraction of the total sub-carriers.
A control channel is dedicated for all the signalling that enables and coordinates the entire CRTI-M scheme.

\subsection{MAC layer co-ordination and distributed implementation}

To make the CRTI-M scheme workable, a MAC layer schedule is needed to co-ordinate the cross-layer activities in the network. Under normal operation, each PU is communicating on its direct link, and the SUs are monitoring the licensed spectrum to detect a transmission opportunity. When a PU detects that its direct link is weak (based on high BER or delayed acknowledgements), it seeks cooperation from the SUs to relay its data. Consequently, the PU $T x_{q}$ sends a cooperation request $\left(C R E Q_{q}\right)$ to the SU network $(q \in \mathbb{Q}$, initially $\mathbb{Q}$ is the set of requesting PUs). It is piggy-backed with the information of the throughput that the PU is experiencing on its weak direct link $\left(C_{\operatorname{dir}_{q}}\right)$. This knowledge is used in computing the time incentive in the given frame. The information is received by the SUs within the radio range of each PU Tx . We design the relaying scheme considering the fact that the PU does not have any cognitive processing capability, and apart from the initial CREQ signaling, the PU should be oblivious to the relaying strategy adopted by the SU network.

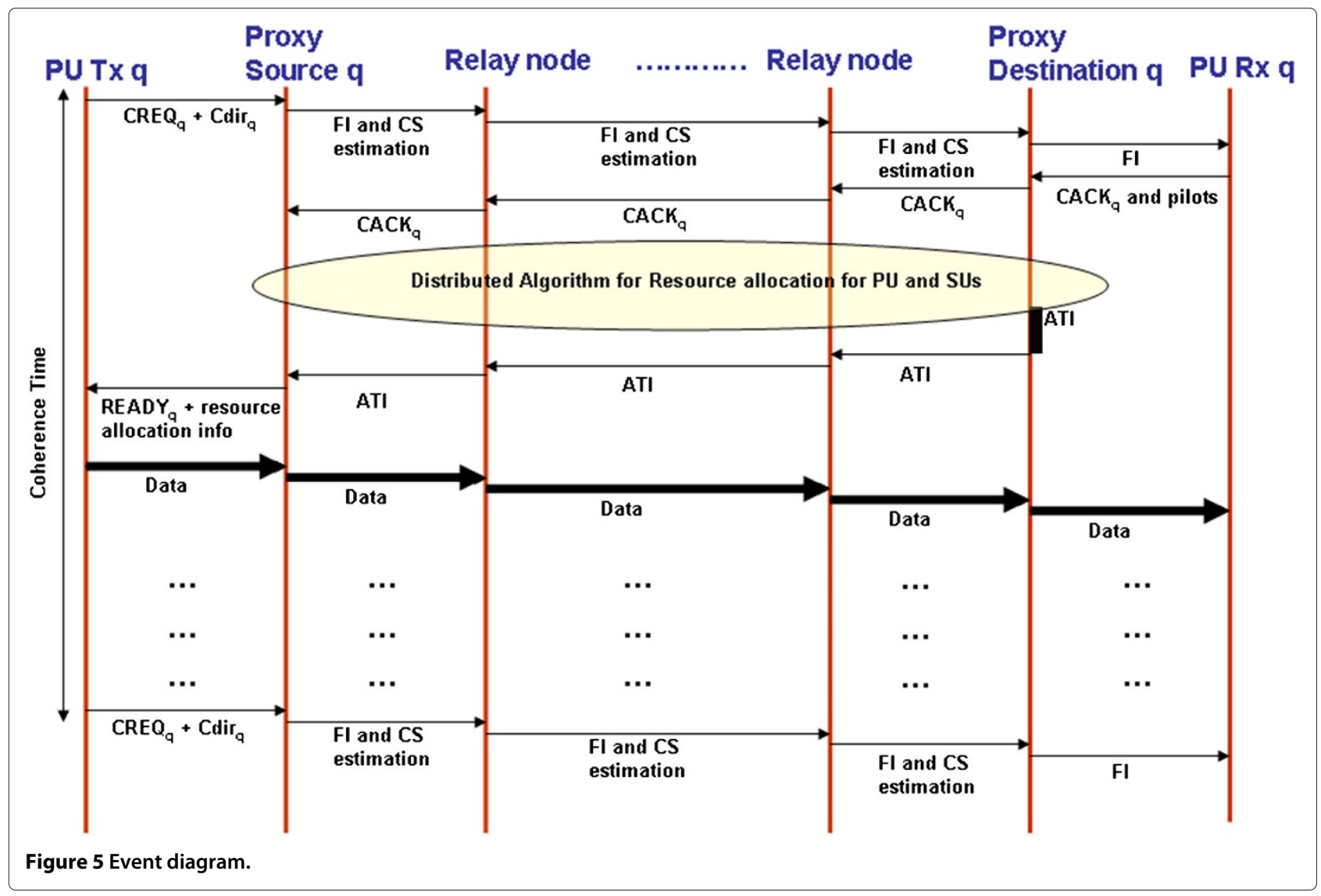




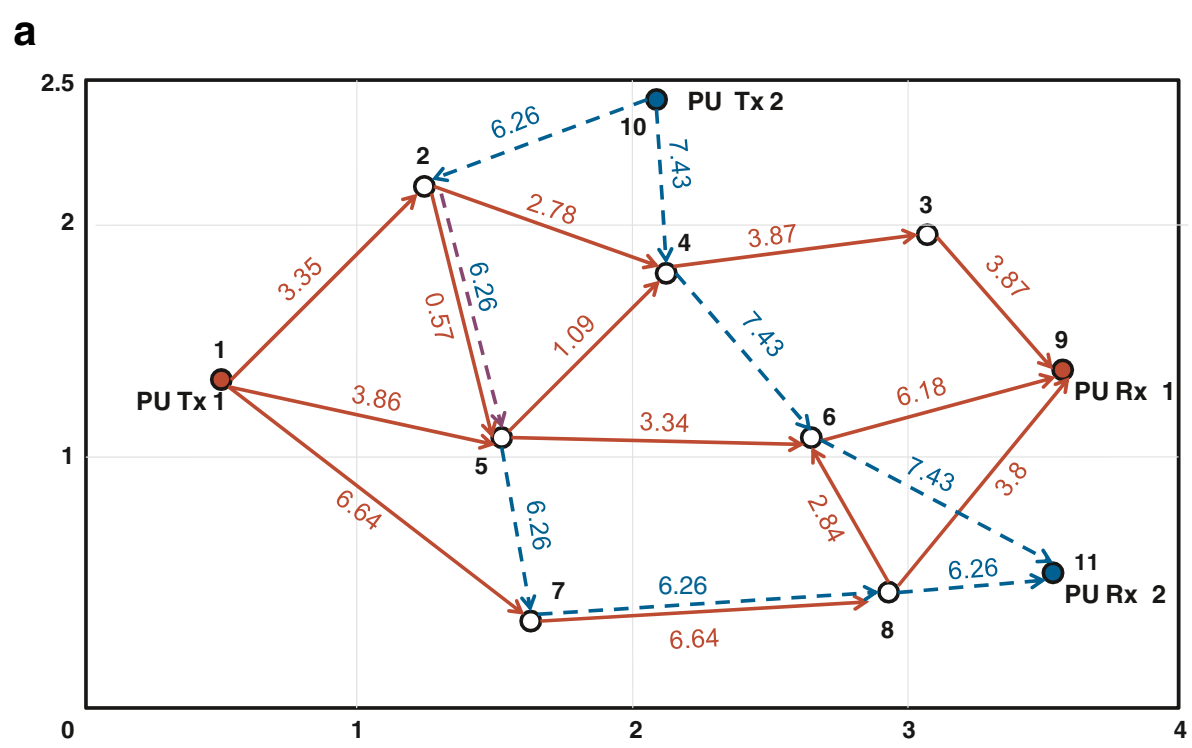

b

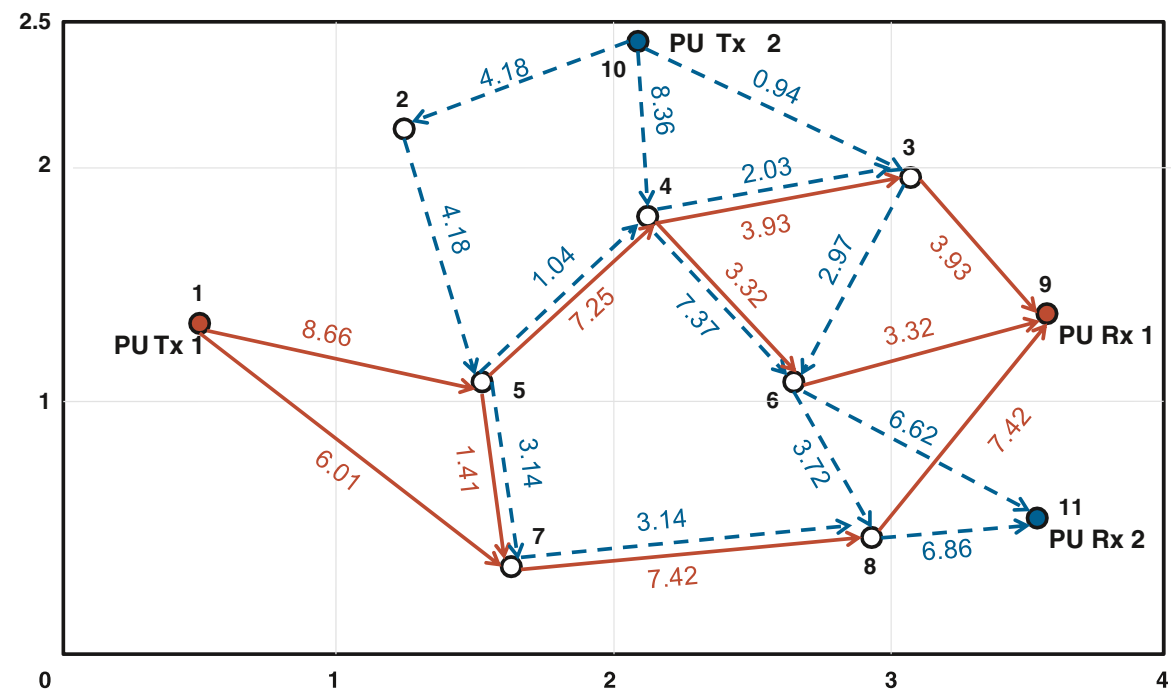

Figure 6 Flow allocation: (a) BRR. (b) ABR.

As such, it is proposed that the nodes which are in the immediate radio range of each PU transmitter act as a proxy source for the SU network (Figure 4b). CREQs from multiple PUs may be received in the control interval of the frame (the MAC frames will be explained subsequently). Those received in the data interval will be queued to be serviced in the control interval of the next frame. Upon receiving the $C R E Q_{q}$, the proxy source nodes for each PU transceiver form a cluster and designate a cluster-head $P S_{q}$. Each $P S_{q}$ sends a frame initialization (FI) command which is propagated throughout the $\mathrm{SU}$ network to indicate the beginning of the symbiotic relaying. This is followed by local channel state estimation at each node.
We propose that the nodes of the last hop, which terminate in the PU $R x_{q}$, form a cluster called the proxy destination to shield the PU $R x_{q}$ from the operation of the SU network (Figure $4 \mathrm{~b}$ ). On receiving the $F I$, the PU $R x_{q}$ responds by sending an Acknowledgement for the Cooperation Request $\left(C A C K_{q}\right)$ and pilots so that its channel can be estimated by the proxy destination nodes. The $C A C K_{q}$ propagates through the network and reaches the proxy source nodes to indicate that the network is ready for CRTI-M.

Each SU node now executes the distributed algorithm (either $B R R$ or $A B R$ ) for resource allocation. The algorithm is executed simultaneously and independently for each PUs' session. Periodic exchange of beacons with its 
Table 1 Results for $B R R$

\begin{tabular}{|c|c|c|}
\hline $\begin{array}{l}\text { Edge } \\
(i, j)\end{array}$ & $\begin{array}{l}\text { Frequency band } \\
x_{i j}^{(m)}\end{array}$ & $\begin{array}{l}\text { Power (W) } \\
P_{i j}^{(m)}\end{array}$ \\
\hline$(1,2)$ & 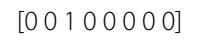 & 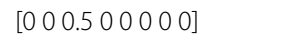 \\
\hline$(1,5)$ & 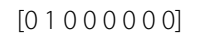 & 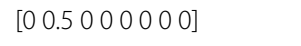 \\
\hline$(1,7)$ & 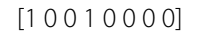 & {$\left[\begin{array}{llllllllllllll}0.5 & 0 & 0 & 0.5 & 0 & 0 & 0 & 0\end{array}\right]$} \\
\hline$(2,4)$ & 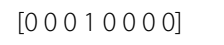 & 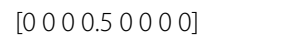 \\
\hline$(2,5)$ & 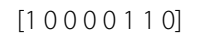 & 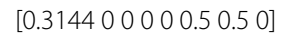 \\
\hline$(3,9)$ & {$\left[\begin{array}{llllllll}1 & 0 & 0 & 0 & 0 & 0 & 0 & 0\end{array}\right]$} & 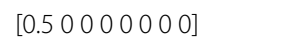 \\
\hline$(4,3)$ & 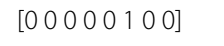 & 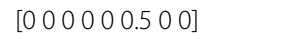 \\
\hline$(4,6)$ & {$\left[\begin{array}{lllllllll}0 & 0 & 0 & 0 & 1 & 0 & 0 & 1\end{array}\right]$} & 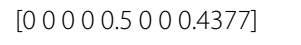 \\
\hline$(5,4)$ & {$\left[\begin{array}{llllllll}0 & 0 & 1 & 0 & 0 & 0 & 0 & 0\end{array}\right]$} & 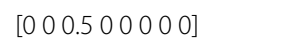 \\
\hline$(5,6)$ & 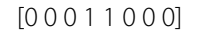 & {$\left[\begin{array}{lllllllllllllll}0 & 0 & 0.5 & 0.1091 & 0 & 0 & 0\end{array}\right]$} \\
\hline$(5,7)$ & 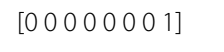 & {$\left[\begin{array}{lllllllllllll}0 & 0 & 0 & 0 & 0 & 0 & 0 & 0.4026\end{array}\right]$} \\
\hline$(6,9)$ & 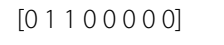 & {$\left[\begin{array}{llllllllllllll}0 & 0.5 & 0.5 & 0 & 0 & 0 & 0 & 0\end{array}\right]$} \\
\hline$(6,11)$ & 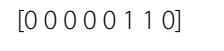 & {$\left[\begin{array}{llllllll}0 & 0 & 0 & 0 & 0 & 0.5 & 0.5 & 0\end{array}\right]$} \\
\hline$(7,8)$ & {$\left[\begin{array}{lllllllll}0 & 1 & 1 & 0 & 0 & 1 & 1 & 0\end{array}\right]$} & 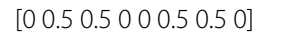 \\
\hline$(8,6)$ & 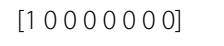 & 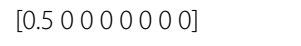 \\
\hline$(8,9)$ & 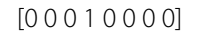 & 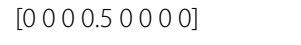 \\
\hline$(8,11)$ & 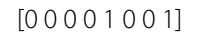 & 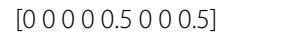 \\
\hline$(10,2)$ & {$\left[\begin{array}{llllllllll}0 & 0 & 0 & 0 & 1 & 0 & 0 & 1\end{array}\right]$} & 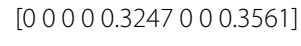 \\
\hline$(10,4)$ & {$\left[\begin{array}{lllllllll}0 & 0 & 0 & 0 & 0 & 1 & 1 & 0\end{array}\right]$} & {$\left[\begin{array}{llllllll}0 & 0 & 0 & 0 & 0 & 0.5 & 0.5 & 0\end{array}\right]$} \\
\hline
\end{tabular}

neighbors is required at each node to execute the modules described earlier. It is only when a node is shared by multiple sessions, that a conflict in the resource allocation arises. The common node resolves the conflict by giving preference to the session $q \in \mathbb{Q}$ with the highest ratio of $\operatorname{mcap}(q)$ to $C_{\mathrm{dir}_{q}}$.

The proxy destination nodes for each PU receiver also designate a cluster-head $P D_{q}$. They are designated to play two important roles: (i) They sum the mcap of every iteration of the corresponding PU session, which aides the determination of the final $C_{\text {rel }_{q}}$, and consequently the time incentive $\lambda_{t_{q}}$; (ii) if $C_{\mathrm{rel}_{q}}<C_{\operatorname{dir}_{q}}$ for any $q \in \mathbb{Q}$, that PU is eliminated from participating in CRTI-M and the algorithm is repeated for the others; and (iii) they track the algorithm termination, in which case the Algorithm Termination Information (ATI) propagates on the reverse paths towards the proxy source nodes, which in turn inform the corresponding PU $T x_{q}$ to start data transmission on the allocated resources by means of the READY signal. The complete event diagram with respect to each $\mathrm{PU}$ is depicted in Figure 5.

The proposed MAC scheduling protocol is meant to provide a unified framework for both the entities of the CRTI-M scheme, viz. the PU and SU. If an SU has its own data to transmit in the time incentive, these requests are captured in the control interval and the distributed algorithm for their sum throughput maximization is also executed. Then in the data interval, the PU and SUs data are time division multiplexed (Figure 4c). The total time for the control interval is acceptable, provided it is a small fraction of the channel coherence time, which is true in a slow fading environment as is assumed in this study.

\section{Simulation results and discussion}

We have simulated a network with the nodes randomly distributed in an area of $10^{2}$ units as shown in Figure 6a. Nodes 2-8 represent the SU relay nodes. Two PU transceivers have been considered: Nodes 1-9 represent PU T $T x_{1}$-PU $R x_{1}$ and nodes $10-11$ represent PU Tx $x_{2}-$ PU $R x_{2}$. All the links undergo Rayleigh multi-path fading, defined in the time domain by $\sum_{l=0}^{L-1} h_{l} \delta(t-l T)$ where $h_{l}$ is the complex amplitude of path $l$, and $L$ is the number of channel taps. The $l$ th channel coefficient between two nodes with a distance $d$ between them is distributed as $\mathcal{N}\left(0,1 / d^{\eta}\right)$ and the frequency domain channel is given by its Fourier Transform. The path loss exponent $\eta=2.5$. Each PU's band-set comprises four OFDM bands: bands 1-4 for PU 1 and bands 5-8 for PU 2. The OFDM subcarrier bandwidth unit is $\mathrm{Hz}$. The other system parameters are $\sigma^{2}=10^{-4} \mathrm{~W}, P_{T}=0.01 \mathrm{~W}, P_{I}=0.001 \mathrm{~W}, P_{\text {peak }}=$ $0.5 W, P_{\text {node }_{i}}=2 W$ (it is the same for each node $i$ ).

MATLAB has been used to simulate the environment and to execute the distributed algorithms. The LINGO [19] software has been used to obtain the centralized

Table 2 Results for $A B R$

\begin{tabular}{|c|c|c|}
\hline $\begin{array}{l}\text { Edge } \\
(i, j)\end{array}$ & $\begin{array}{l}\text { Frequency band } \\
x_{i j}^{(m)}\end{array}$ & $\begin{array}{l}\text { Power (W) } \\
P_{i j}^{(m)}\end{array}$ \\
\hline$(1,5)$ & {$\left[\begin{array}{llllllll}0 & 0 & 1 & 1 & 0 & 0 & 0 & 0\end{array}\right]$} & {$\left[\begin{array}{llllllllllll}0 & 0 & 0.5 & 0.5 & 0 & 0 & 0 & 0\end{array}\right]$} \\
\hline$(1,7)$ & {$\left[\begin{array}{llllllllllll}1 & 1 & 0 & 0 & 0 & 0 & 0 & 0\end{array}\right]$} & {$\left[\begin{array}{llllllllll}0.5 & 0.5 & 0 & 0 & 0 & 0 & 0 & 0\end{array}\right]$} \\
\hline$(2,5)$ & {$\left[\begin{array}{llllllll}1 & 0 & 0 & 0 & 0 & 0 & 1 & 0\end{array}\right]$} & 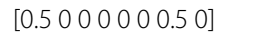 \\
\hline$(3,6)$ & {$\left[\begin{array}{lllllllll}0 & 0 & 0 & 0 & 0 & 0 & 1 & 0\end{array}\right]$} & {$\left[\begin{array}{lllllllll}0 & 0 & 0 & 0 & 0 & 0 & 0.5 & 0\end{array}\right]$} \\
\hline$(3,9)$ & {$\left[\begin{array}{lllllllll}0 & 1 & 0 & 0 & 0 & 0 & 0 & 0\end{array}\right]$} & {$\left[\begin{array}{lllllllllllll}0 & 0.5 & 0 & 0 & 0 & 0 & 0 & 0\end{array}\right]$} \\
\hline$(4,3)$ & {$\left[\begin{array}{lllllllll}1 & 0 & 0 & 0 & 0 & 0 & 0 & 0\end{array}\right]$} & {$\left[\begin{array}{lllllllllll}0.5 & 0 & 0 & 0 & 0 & 0 & 0 & 0\end{array}\right]$} \\
\hline$(4,6)$ & {$\left[\begin{array}{lllllllll}0 & 0 & 1 & 1 & 0 & 0 & 0 & 0\end{array}\right]$} & {$\left[\begin{array}{llllllll}0 & 0 & 0.5 & 0.5 & 0 & 0 & 0 & 0\end{array}\right]$} \\
\hline$(5,4)$ & {$\left[\begin{array}{lllllllll}0 & 0 & 0 & 0 & 1 & 1 & 0 & 0\end{array}\right]$} & {$\left[\begin{array}{lllllllllllll}0 & 0 & 0 & 0 & 0.5 & 0.5 & 0 & 0\end{array}\right]$} \\
\hline$(5,7)$ & {$\left[\begin{array}{llllllll}0 & 0 & 0 & 0 & 0 & 0 & 0 & 1\end{array}\right]$} & 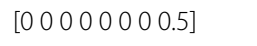 \\
\hline$(6,8)$ & {$\left[\begin{array}{lllllllll}0 & 1 & 0 & 0 & 0 & 0 & 0 & 0\end{array}\right]$} & 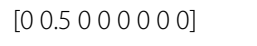 \\
\hline$(6,9)$ & {$\left[\begin{array}{llllllllllll}1 & 0 & 0 & 0 & 0 & 0 & 0 & 0\end{array}\right]$} & {$\left[\begin{array}{lllllllll}0.2994 & 0 & 0 & 0 & 0 & 0 & 0 & 0\end{array}\right]$} \\
\hline$(6,11)$ & {$\left[\begin{array}{llllllll}0 & 0 & 0 & 0 & 1 & 1 & 0 & 0\end{array}\right]$} & {$\left[\begin{array}{llllllll}0 & 0 & 0 & 0 & 0.5 & 0.2863 & 0 & 0\end{array}\right]$} \\
\hline$(7,8)$ & {$\left[\begin{array}{llllllll}0 & 0 & 0 & 0 & 1 & 0 & 0 & 0\end{array}\right]$} & 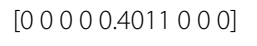 \\
\hline$(8,9)$ & {$\left[\begin{array}{lllllllll}0 & 0 & 1 & 1 & 0 & 0 & 0 & 0\end{array}\right]$} & {$\left[\begin{array}{llllllll}0 & 0 & 0.5 & 0.5 & 0 & 0 & 0 & 0\end{array}\right]$} \\
\hline$(8,11)$ & {$\left[\begin{array}{llllllll}0 & 0 & 0 & 0 & 0 & 0 & 1 & 1\end{array}\right]$} & {$\left[\begin{array}{llllllll}0 & 0 & 0 & 0 & 0 & 0 & 0.5 & 0.5\end{array}\right]$} \\
\hline$(10,2)$ & 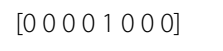 & 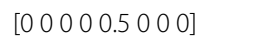 \\
\hline$(10,3)$ & {$\left[\begin{array}{llllllll}0 & 0 & 0 & 0 & 0 & 1 & 0 & 0\end{array}\right]$} & 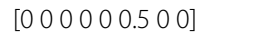 \\
\hline$(10,4)$ & {$\left[\begin{array}{llllllll}0 & 0 & 0 & 0 & 0 & 0 & 1 & 1\end{array}\right]$} & {$\left[\begin{array}{llllllll}0 & 0 & 0 & 0 & 0 & 0 & 0.5 & 0.5\end{array}\right]$} \\
\hline
\end{tabular}


solutions to the $B R R$ and $A B R$ optimization problems. LINGO deploys the branch-and-bound technique to tackle the integer variables $x_{i j}^{(m)}$, and its nonlinear solver employs successive linear programming at each branch. For an extremely small network (one-hop, and two bands on each link), the results obtained from LINGO have been theoretically verified using the above techniques, though not included in the article. But for the network assumed in the simulation, the combinatorial nature of the problems makes it computationally prohibitive to do so.

We first report the results for the centralized solution of the $B R R$ problem, for a single instance of the channel conditions. The flow splitting and flow conservation are depicted in Figure $6 \mathrm{a}$, where the numbers denote the flow in bits/s/Hz. The maximized sum throughput of the two PUs' sessions is obtained as $27.54 \mathrm{bits} / \mathrm{s} / \mathrm{Hz}$. Table 1 reports the corresponding band assignment and power allocation. It can be observed that the session of PU $T x_{1}-$ PU $R x_{1}$ always occupies its own band-set, i.e., $\{1,2,3,4\}$, while the session of PU $T x_{2}-$ PU $R x_{2}$ occupies its band-set $\{5,6,7,8\}$.

The results for $A B R$, for the same channel conditions as those used above, are reported in Figure $6 \mathrm{~b}$ and Table 2. The maximized sum throughput of the PUs' sessions is obtained as $28.15 \mathrm{bits} / \mathrm{s} / \mathrm{Hz}$. It can be observed that the sessions occupy the complete available band-set, i.e., $\{1-8\}$ within the SU network, but from PU $T x_{q}$ to the SU network and the SU network to PU $R x_{q}$, only the respective band sets are used.

In Figure 7, we report the cumulative time incentive obtained in a unit time slot, i.e., $\sum_{q} \lambda_{t_{q}}$, by varying the number of PU transceivers. $C_{\operatorname{dir}_{q}}$ was assumed as $4 \mathrm{bits} / \mathrm{s} / \mathrm{Hz}$ for each PU, and $C_{\mathrm{rel}_{q}}$ was obtained from the centralized cross-layer optimization results. As expected,
$A B R$ provides a higher time incentive as compared to $B R R$. Also, the difference in the incentive obtained from both schemes increases with the number of users. This is due to the fact that each participating PU provides its own bandwidth for use by all other PUs during the relaying process in $A B R$, which results in greater channel diversity as compared to $B R R$. These results were obtained by adding PUs randomly to the topology described earlier; they have been averaged over three different positions of nodes in the network (in the given area of $10^{2}$ units), with 10 independent channel realizations in each case, i.e., totally 30 instances. We have been unable to incorporate any more than 4 users, as the constraint and variable limitation in the version of LINGO used made it computationally prohibitive. We anticipate that by adding more PUs, a point will be reached beyond which the cumulative time incentive will not increase any further-the power constraint will not allow any further increase in the relay throughput even with the addition of frequency bands. Intuitively, this saturation will be reached earlier for $B R R$ as compared to $A B R$.

The execution of the proposed distributed algorithm for $B R R$ is depicted iteration-wise (first four iterations) for a single instance of the channel and two PU transceivers, in Figure $8 \mathrm{a}-\mathrm{d}$. It can be observed that on execution of the algorithm, first a path is formed between PU $T x_{1}-\mathrm{PU} R x_{1}$. The bands used on each link and the corresponding flow (bits/s/Hz) are indicated in the figure. In the third iteration, another physical path is formed between the same source-destination pair. In the fourth iteration, a path is formed for the second session, i.e., PU T $T x_{2}-\mathrm{PU} R x_{2}$. The final allocation is shown in Figure 8e. The corresponding power allocation and band assignment are documented in Table 3.

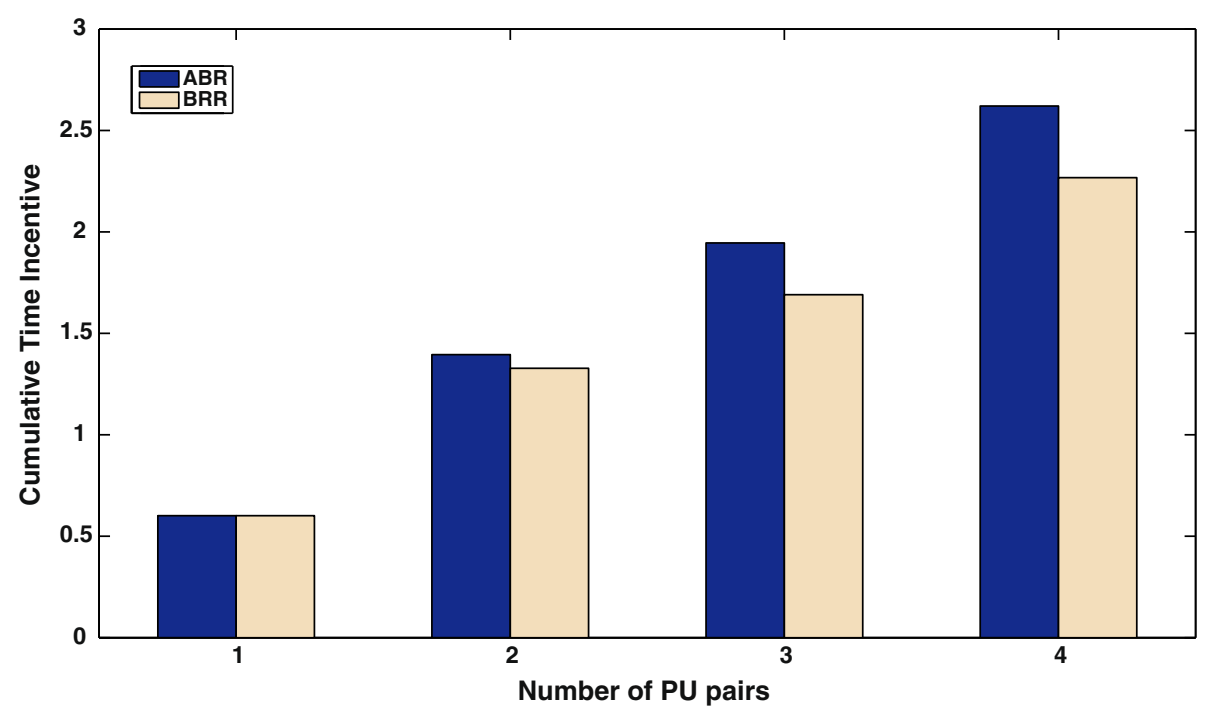

Figure 7 Cumulative time incentive versus number of PUs. 

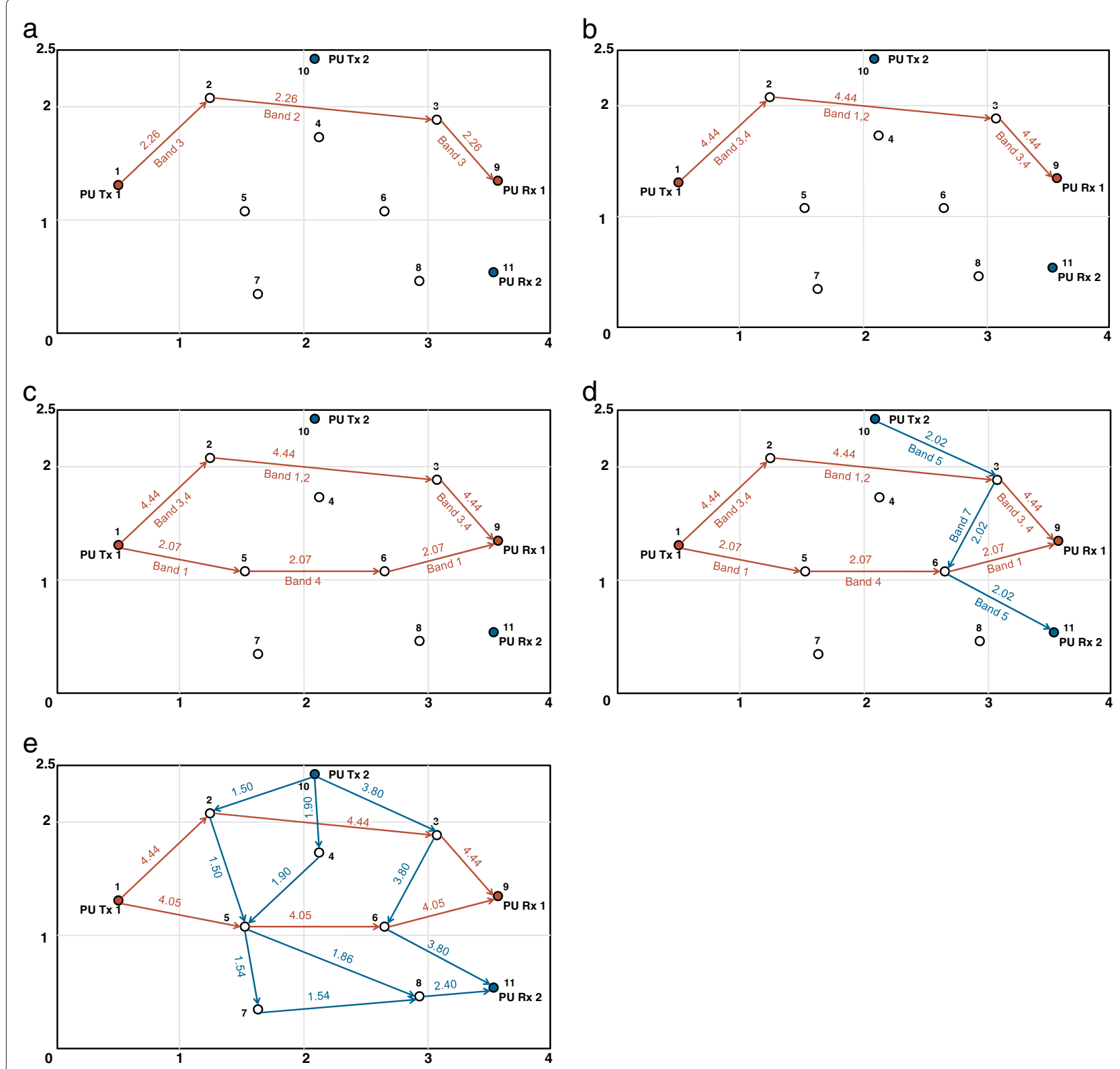

Figure 8 Execution of distributed BRR algorithm: (a) Iteration 1. (b) Iteration 2. (c) Iteration 3. (d) Iteration 4. (e) Final.

Similar results will be observed on executing the distributed $A B R$ algorithm.

Next, we plot the cumulative time incentive for $B R R$ with respect to channel instances, obtained on the execution of the distributed algorithm. It is compared with the upper bound obtained by tangential supports to the $\log$ curve and the feasible centralized solution (Figure 9a). As expected, the centralized solution provides a higher incentive than the distributed algorithm which relies on local information. However, the solution obtained from the distributed algorithm is quite close to the centralized result, for instance, for channel conditions 1 and 6 , the distributed result is 98.07 and $98.01 \%$ of the corresponding upper bound. Which means these results are even closer to the actual solution. On an average, the distributed result is $87.51 \%$ of the centralized solution. These results were plotted for two PU transceivers, and $C_{\operatorname{dir}_{q}}$ was assumed as $4 \mathrm{bits} / \mathrm{s} / \mathrm{Hz}$ for each to compute the time incentive. Similar results are observed for $A B R$, with the distributed result being $92.4 \%$ of the corresponding centralized solution (Figure 9b).

In Figure 10, we compare the distributed results obtained for both the CRTI-M schemes to illustrate the point that $A B R$ performs better than $B R R$ due to the 


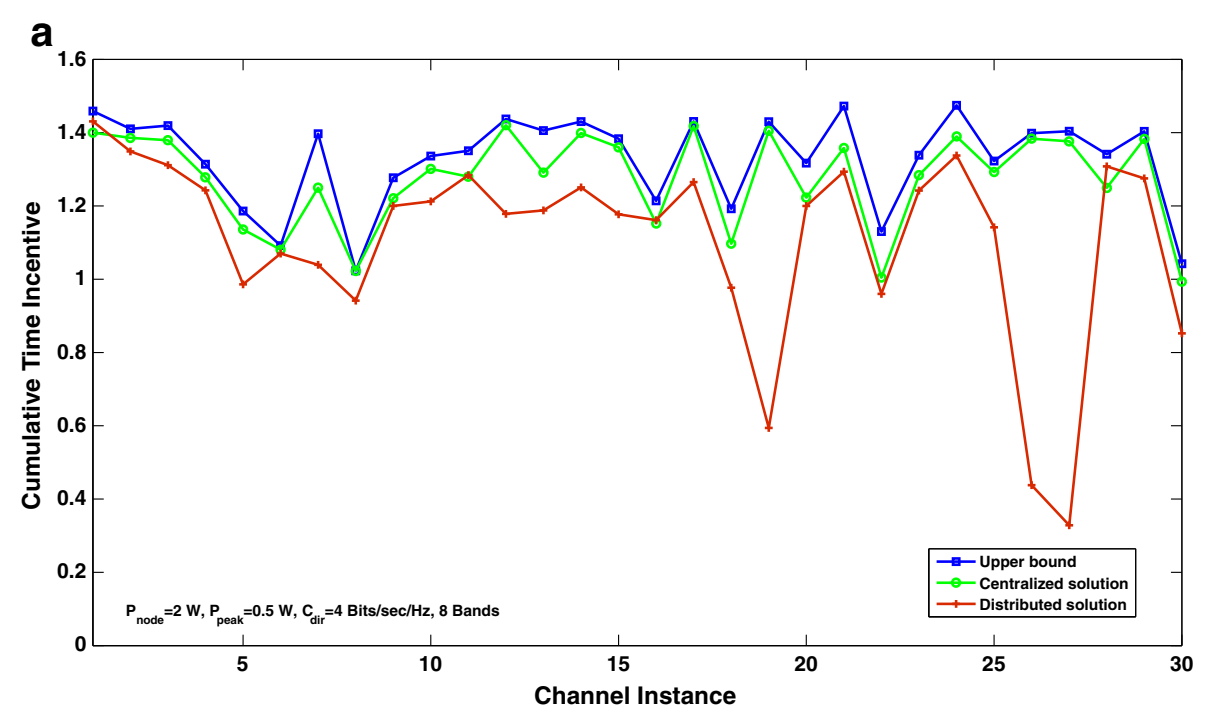

b

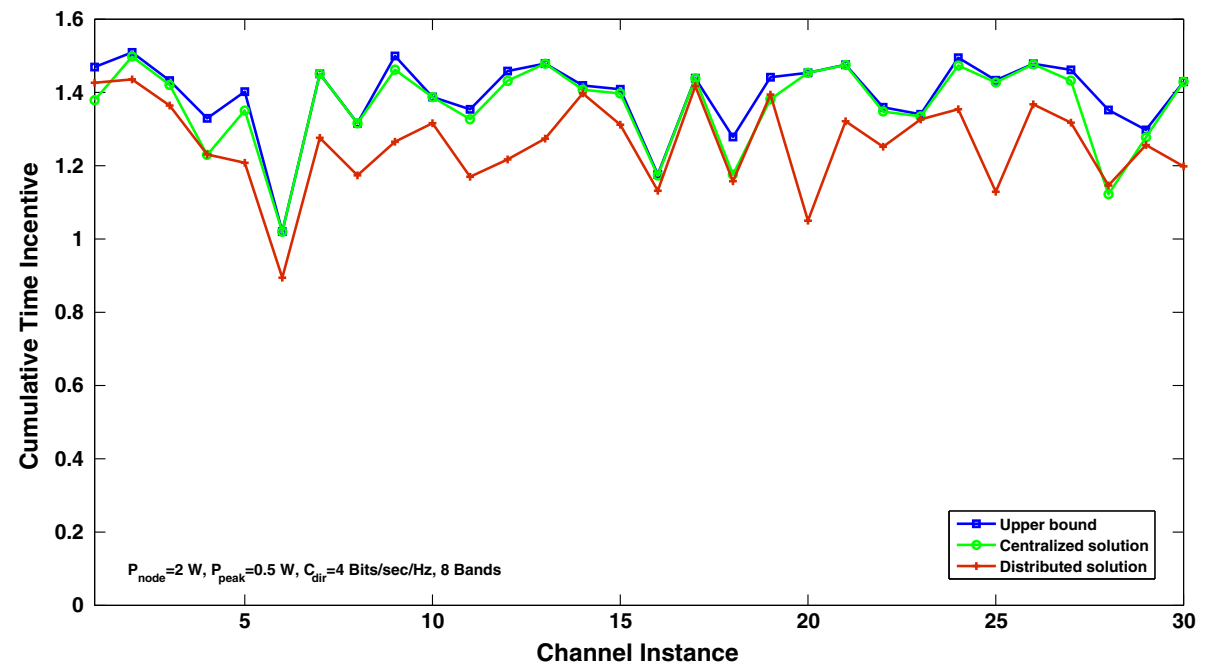

Figure 9 Cumulative time incentive: (a) BRR. (b) ABR.

higher channel diversity it provides. This trend was also observed earlier for the centralized solutions, and further validates the proposed distributed algorithms. On an average, the time incentive obtained from $A B R$ exceeds that obtained from $B R R$. In a few cases, though, the result of $A B R$ is lower than that for $B R R$, which may be attributed to the dominating influence of the channel conditions and the limitations of the distributed algorithms as compared to the centralized.

\section{Related study}

We review the literature in the following contexts, which are relevant to the current work.

\subsection{SCR for CR}

Surrounding the concept of SCR the following models have been proposed: Simeone et al. [3] have used game theoretic tools to analyze the performance of cooperation in a CR network, wherein the PU leases the owned spectrum to an adhoc network of SUs in exchange for cooperation in the form of transmission power from the SUs. The model proposed by Zhang and Zhang [4] is more rational; when the PU's demand is satisfied, it is willing to enhance its benefit in any other format, for instance, by collecting a higher revenue from the SU. Xue et al. [5] have considered a single full-duplex amplify-and-forward (AF) SU relay to assist the PU transmission. Gong et al. [6] have analyzed the power and diversity gains obtained 
Table 3 Results for distributed BRR

\begin{tabular}{|c|c|c|}
\hline $\begin{array}{l}\text { Edge } \\
(i, j)\end{array}$ & $\begin{array}{l}\text { Frequency band } \\
x_{i j}^{(m)}\end{array}$ & $\begin{array}{l}\text { Power (W) } \\
P_{i j}^{(m)}\end{array}$ \\
\hline$(1,2)$ & 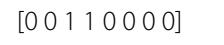 & {$\left[\begin{array}{llllllll}0 & 0 & 0.4511 & 0.3876 & 0 & 0 & 0 & 0\end{array}\right]$} \\
\hline$(1,5)$ & 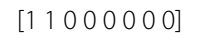 & {$\left[\begin{array}{lllllll}0.2192 & 0.1654 & 0 & 0 & 0 & 0 & 0\end{array}\right]$} \\
\hline$(2,3)$ & 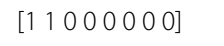 & {$\left[\begin{array}{llllllllllll}0.5 & 0.5 & 0 & 0 & 0 & 0 & 0 & 0\end{array}\right]$} \\
\hline$(2,5)$ & 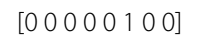 & 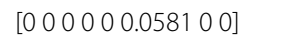 \\
\hline$(3,6)$ & 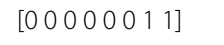 & 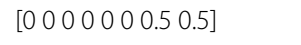 \\
\hline$(3,9)$ & 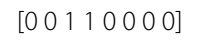 & {$\left[\begin{array}{llllllll}0 & 0 & 0.1645 & 0.1444 & 0 & 0 & 0 & 0\end{array}\right]$} \\
\hline$(4,5)$ & 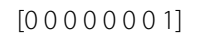 & {$\left[\begin{array}{llllllllllllll}0 & 0 & 0 & 0 & 0 & 0 & 0 & 0.0688\end{array}\right]$} \\
\hline$(5,6)$ & 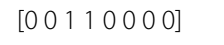 & {$\left[\begin{array}{llllllllllllll}0 & 0 & 0.5 & 0.5 & 0 & 0 & 0 & 0\end{array}\right]$} \\
\hline$(5,7)$ & 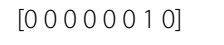 & 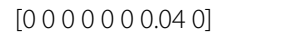 \\
\hline$(5,8)$ & 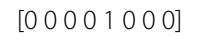 & 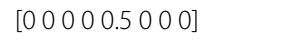 \\
\hline$(6,9)$ & 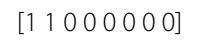 & {$\left[\begin{array}{lllllllll}0.5 & 0.5 & 0 & 0 & 0 & 0 & 0 & 0\end{array}\right]$} \\
\hline$(6,11)$ & 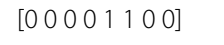 & {$\left[\begin{array}{llllllll}0 & 0 & 0 & 0 & 0.4471 & 0.2961 & 0 & 0\end{array}\right]$} \\
\hline$(7,8)$ & 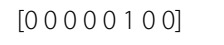 & 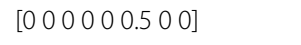 \\
\hline$(8,11)$ & {$\left[\begin{array}{lllllllll}0 & 0 & 0 & 0 & 0 & 0 & 1 & 1\end{array}\right]$} & {$\left[\begin{array}{llllllll}0 & 0 & 0 & 0 & 0 & 0 & 0.1853 & 0.1224\end{array}\right]$} \\
\hline$(10,2)$ & {$\left[\begin{array}{lllllllll}0 & 0 & 0 & 0 & 0 & 0 & 0 & 1\end{array}\right]$} & 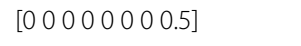 \\
\hline$(10,3)$ & 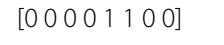 & 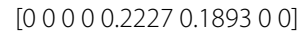 \\
\hline$(10,4)$ & {$\left[\begin{array}{llllllll}0 & 0 & 0 & 0 & 0 & 0 & 1 & 0\end{array}\right]$} & {$\left[\begin{array}{lllllllllllll}0 & 0 & 0 & 0 & 0 & 0 & 0.2333 & 0\end{array}\right]$} \\
\hline
\end{tabular}

by AF relaying of the PU's data by multiple cooperating SUs. All of the aforementioned works in literature have considered either a single relay node or single channel CR networks. The authors have also contributed significantly towards $S C R$ paradigms for multi-channel multihop networks [7-11], the details of which are provided in (1) section.

\subsection{Cross-layer optimization for multi-channel networks} Cross-layer optimization problems have received considerable attention in literature ([14-22] and the references therein). Shi et al. [14] have addressed power control, frequency band scheduling, and flow routing for the physical model in a multi-hop multi-channel CR network with the objective of maximizing the rates of a set of user communication sessions. The power is quantized to a finite number of levels, and a centralized solution is developed using the branch-and-bound framework. Ma and Tsang [21] use a similar model but a simplistic problem formulation, with the assumption of uniform power and only band assignment and flow routing as the variables. The formulation of Zhang et al. [16] aims at minimizing the transmission time for the user sessions by joint consideration of spectrum allocation, routing, and time scheduling. Besides these, there also exist cross-layer formulations for conventional multi-channel wireless networks with all or some of the flow, scheduling, power constraints, typically with the objective of maximizing users' throughput, or minimizing power consumption [22-24].

\subsection{Distributed algorithms for cross-layer formulations}

The work pertaining to distributed algorithms for crosslayer optimization problems is limited. Lin and Shroff [25] consider maximizing the sum of the users' utility functions, assuming a single path for each user and a single channel network, to determine the rate and link schedule. Palomar and Chiang [26] also formulate a similar problem within a power constrained situation, which they address using different decomposition techniques. Lin and Rasool [27] have developed a distributed algorithm that jointly

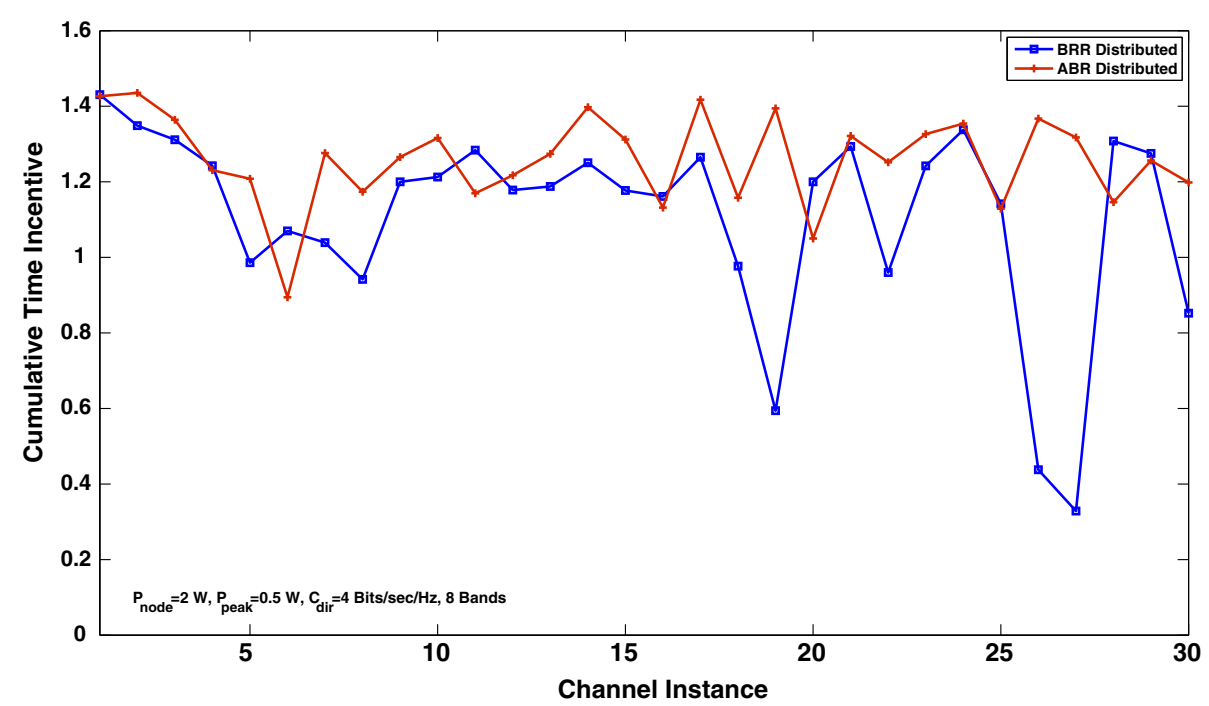

Figure 10 Comparing BRR and ABR. 
solves the channel-assignment, time scheduling and routing problem to maximize the system capacity, with fixed power, in a multi-channel network. Joint allocation of power, frequency bands, and routing in a multi-channel multi-hop network in a distributed manner is more challenging. The centralized framework suggested by Shi et al. [28] is quite similar to ours, hence we feel the need to provide a detailed comparison with the former. The main difference in the cross-layer formulations is that this study incorporates the requirements of $B R R$ (each PU relaying on its own band) and $A B R$ (each PU utilizing all the available bands), while that of Shi et al. is concerned with throughput maximization of SUs' sessions. Also, we consider the sum interference from all the neighboring nodes for power allocation. The distributed algorithm described by Shi et al. differ from ours in the following aspects: (i) the metric used by the former for path selection is bandwidth footprint product which targets optimal spatial occupancy, whereas the authors use a metric which gives weightage to both the net throughput that can be obtained from a path and also that link which may pose a bottleneck, which seems more appropriate in a throughput maximization scenario; (ii) the former's strategy involves a Conservative Iteration Process, which allocates resources for the sessions and an Aggressive Iteration Process, which tries to increase the throughput of sessions with lower throughput (lower scaling factors) by releasing the allocated resources of the others with higher throughput. The authors, on the other hand, propose that on encountering paths from multiple sessions, a common node will instantaneously resolve the conflict by giving preference to that session which is likely to derive a higher benefit-this approach befits a distributed implementation in terms of practical execution and time; (iii) given the fact that the source and destination are PUs, while the relay nodes are SUs, the exchange of information between the two entities is rather restricted. In this study, the detailed MAC schedule is provided, which will address this issue while facilitating the distributed algorithm for resource allocation. In the work of Shi et al., there is no such concern since only one entity, viz. the SU is involved in the process; (iv) the algorithm of Shi et al. allocates power on unused bands based on the detection threshold which results in the same metric for all bands irrespective of the channel gains. On the other hand, the proposed algorithm operates on the peak power, which gives importance to the gains when selecting high throughput paths. Moreover, the proposed algorithm is more meticulous in meeting the constraints of the centralized problem, and has specialized modules to account for $B R R$ and $A B R$.

\section{Conclusion}

The main focus of this article is to develop distributed algorithms for the two methods of CRTI-M, viz. BRR and
$A B R$. $A B R$ which allows a PU to use all other PUs' bands when relaying through the $\mathrm{SU}$ network provides a higher cumulative time incentive for the SUs. BRR, on the other hand, which allows every PU to only use its own band, makes management and PU prioritization easier. The proposed distributed algorithms are meticulous in meeting the constraints of the corresponding centralized problems and the results are in close proximity of the centralized results. A MAC scheduling protocol is described which gives emphasis to the distributed implementation and presents a unified framework for the PUs and SUs.

Competing interests

The authors declare that they have no competing interests.

\section{Acknowledgements}

This study was supported by the Ministry of Communication and Information Technology, Government of India, New Delhi. The study was also supported by the Microsoft Corporation and Microsoft Research India under the Microsoft Research India PhD Fellowship Award 2009. A small part of this study (only the centralized problem formulation) has been presented at IEEE VTC Fall 2011.

\section{Author details}

${ }^{1}$ Indian Institute of Technology Bombay, Mumbai 400076, India. ${ }^{2}$ Indian Institute of Technology Hyderabad, Hyderabad 502205, India.

Received: 9 February 2012 Accepted: 28 September 2012 Published: 2 November 2012

\section{References}

1. S Haykin, Cognitive radio: brain-empowered wireless communications IEEE Trans. Sel. Areas Commun. 23, 201-220 (2005)

2. H Zhang, Cognitive radio networking for green communications and green spectrum. [Online] Available from http://www.comnets.org/ keynote.html

3. O Simeone, Y Bar-Ness, U Spagnolini, in Cooperative Wireless Communications, ed. by Y Zhang, $\mathrm{H}$ Chen, and M Guizani. Cooperative cognitive radio (Auerbach Publications, CRC Press, Boca Raton, 2009), pp. 209-230

4. J Zhang, Q Zhang, in Proc. of ACM MobiHoc, Stackelberg game for utility-based cooperative cognitive radio networks (2009), pp. 23-32

5. P Xue, P Gong, N Cao, DK Kim, in Proc. of KICS the 18th, JCCI, Symbiotic architecture for the cognitive radio networks with amplify-and-forward relaying cooperation (Jeju, Korea, 2009), pp. 49-54

6. P Gong, JH Park, JM Yoo, Y Bo-Sun, DK Kim, in Proc. of 4th International Symposium on Wireless Pervasive Computing, Throughput maximization with multiuser non-selfish cognitive relaying in CR networks (2009), pp. $1-5$

7. T Nadkar, V Thumar, UB Desai, SN Merchant, Symbiotic cooperative relaying in cognitive radio networks with time and frequency incentive. Springer Telecommun. Syst. J.: Special Issue on Mobile Computing and Networking Technologies. doi:10.1007/s11235-011-9494-4

8. T Nadkar, VM Thumar, UB Desai, SN Merchant, in Proc. of IEEE Symp. on New Frontiers in Dynamic Spectrum Access Networks (DySPAN), A cross-layer framework for symbiotic relaying in cognitive radio networks (2011), pp. 498-509

9. TNadkar, VM Thumar, G Shenoy, UB Desai, SN Merchant, in Proc. of the IEEE Global Telecommunications Conference, IEEE GLOBECOM, Cognitive relaying with frequency incentive (2011), pp. 1-6

10. T Nadkar, VM Thumar, G Shenoy, UB Desai, SN Merchant, in Proc. of IEEE Symposium on Personal, Indoor and Mobile Radio Communications (PIMRC), Cognitive relaying with time incentive:protocol design for multiple primary users (2011), pp. 577-582

11. VM Thumar, T Nadkar, G Shenoy, UB Desai, SN Merchant, in Proc. of IEEE Vehicular Technology Conference (VTC), Cognitive relaying with time incentive: multiple primary users (2011), pp. 1-5

12. JM Peha, Approaches to spectrum sharing. IEEE Commun. Mag. 43(2), 10-12 (2005) 
13. W Lehr, J Crowcroft, in Proc. of First IEEE Symp. New Frontiers in Dynamic Spectrum Access Networks, Managing shared access to a spectrum commons (2005), pp. 420-444

14. Y Shi, Y Hou, S Kompella, in Proc. of IEEE MILCOM'08, A cross-layer approach to multi-hop networking with cognitive radios (2008), pp. 1-7

15. TM Cover, Thomas JA, Elements of Information Theory, 2nd edn. (Wiley-Interscience, New York, 2006)

16. J Zhang, Z Zhang, H Luo, A Huang, in Proc. of the IEEE Global Telecommunications Conference, IEEE GLOBECOM, A column generation approach for spectrum allocation in cognitive wireless mesh network (2008), pp. 1-5

17. PA Humblet, An adaptive distributed, Dijkstra shortest path algorithm. Technical Report CICS-P-60, Center for Intelligent Control Systems, MIT, (1981)

18. TH Cormen, CE Leiserson, RL Rivest, C Stein, 2nd edn. (MIT Press and McGraw-Hill, 2001), pp. 651-664

19. LINGO: User's guide (LINDO Systems Inc., 2006)

20. DP Bertsekas, Network Optimization-Continuous and Discrete models (Athena Scientific, Belmont, MA)

21. M Ma, DHK Tsang, in Proc. of 5th IEEE Consumer Communications and Networking Conference CCNC, Joint spectrum sharing and fair routing in cognitive radio networks (2008), pp. 978-982

22. R Bhatia, MS Kodialam, in Proc. of IEEE INFOCOM, On power efficient communication over multi-hop wireless networks: joint routing scheduling and power control (2004), pp. 1457-1466

23. M Alicherry, R Bhatia, L Erran Li, Joint channel assignment and routing for throughput optimization in multiradio wireless mesh networks. IEEE J. Sel. Areas Commun. 24(11), 1960-1971 (2006)

24. K Karakayali, JH Kang, M Kodialam, K Balachandran, in Proc. of IEEE ICC, Joint resource allocation and routing for OFDMA-based broadband wireless mesh networks (2007), pp. 5088-5092

25. X Lin, NB Shroff, in Proc. IEEE Infocom, The impact of imperfect scheduling on cross-layer rate control in wireless networks Miami, 2005), pp. 1804-1814

26. DP Palomar, M Chiang, in Proc. IEEE Infocom, Alternative decompositions for distributed maximization of network utility: framework and applications Spain, 2006), pp. 1-13

27. X Lin, S Rasool, in Proc. IEEE Infocom, A distributed joint channel-assignment, scheduling and routing algorithm for multi-channel ad hoc wireless networks Anchorage, 2007), pp. 1118-1126

28. Y Shi, Y Thomas Hou, H Zhou, S Midkiff, Distributed cross-layer optimization for cognitive radio networks. IEEE Trans. Veh. Technol. 59(8), 4058-4069 (2010)

\section{Submit your manuscript to a SpringerOpen ${ }^{\odot}$ journal and benefit from:}

- Convenient online submission

- Rigorous peer review

- Immediate publication on acceptance

- Open access: articles freely available online

- High visibility within the field

- Retaining the copyright to your article 